\title{
Lista preliminar das plantas alimentícias nativas de Mato Grosso do Sul, Brasil
}

\author{
leda Maria Bortolotto, Geraldo Alves Damasceno-Junior \& Arnildo Pott
}

Fundação Universidade Federal de Mato Grosso do Sul, Instituto de Biociências, Laboratório de Botânica. Bairro Universitário, CEP 79070-900, Campo Grande, Mato Grosso do Sul. iedamaria.bortolotto@gmail.com

Recebido em 27.IX.2014

Aceito em 17.V.2016

DOI 10.21826/2446-8231201873s101

RESUMO - Apresentamos o inventário preliminar das plantas alimentícias silvestres do Mato Grosso do Sul usadas na dieta humana ou com potencial para uso. Incluímos espécies que constam em publicações e em trabalhos inéditos dos autores, cujas coletas, realizadas no estado, estão incorporadas nos herbários CGMS, COR e CPAP. Adicionalmente, foram incluídas espécies de Arecaceae, coletadas no estado depositadas em outros Herbários e espécies dos gêneros Arachis, Dioscorea e Passiflora que constam na Lista de Espécies da Flora do Brasil para o Mato Grosso do Sul. Foram encontradas 294 espécies, distribuídas em 160 gêneros e 67 famílias botânicas. As famílias mais ricas foram Fabaceae (49) e Myrtaceae (38), seguidas por Arecaceae (32) e Passifloraceae (12). Esta é a primeira listagem de espécies alimentícias do estado.

Palavras chaves: frutos comestíveis, Cerrado, Pantanal

ABSTRACT - Preliminary list of native food plants of Mato Grosso do Sul, Brazil - We present a preliminary inventory of wild food plants found in Mato Grosso do Sul that are used in human diet or potentially useful. Species were compiled from publications and from data collected by the authors; specimens deposited in CGMS, COR and CPAP herbaria were also included. Additionally we included species of Arecaceae and of Arachis, Dioscorea and Passiflora cited in the species list of Brazilian flora. We found a total of 294 species distributed in 160 genera and 67 families. The families with highest number of species were Fabaceae (49), Myrtaceae (38), Arecaceae (32) and Passifloraceae (12). This is the first list of edible species for Mato Grosso do Sul.

Keywords: Cerrado, edible fruits, Pantanal

\section{INTRODUÇÃO}

Mato Grosso do Sul possui diversas fisionomias vegetais com uma extensa área ocupada pelos cerrados, representantes da flora chaquenha, além de campos inundáveis, florestas estacionais deciduais e semideciduais, dentre outras (Silva et al. 2011, Pott et al. 2011). As plantas alimentícias nativas (não cultivadas) encontradas nessas fisionomias vegetais têm um papel importante para as populações humanas locais (Bortolotto \& Amorozo 2012), para a fauna silvestre (Pott \& Pott 1994) e para os animais domésticos. Recentemente algumas espécies têm adquirido valor econômico no estado em decorrência do crescente interesse por parte do mercado e incentivadas por projetos que visam a melhoria da qualidade de vida dos moradores de pequenas comunidades locais e a conservação dos recursos (Damasceno-Junior et al.2010).

As primeiras informações sobre plantas alimentícias usadas na dieta humana no atual território sul-matogrossense foram registradas por colonizadores europeus no século XVI, como Cabeza de Vaca, que mencionou espécies alimentícias usadas pelos povos indígenas (Cabeza de Vaca 1987). Nesses relatos, as plantas não eram apresentadas com nomes científicos, tornando incerta a identificação a partir dos nomes populares ou indígenas. Nos séculos XVIII e XIX, viajantes naturalistas contribuíram com informações sobre os costumes e recursos naturais dos povos indígenas (Leite 1995) e com relatos sobre hábitos alimentares no Pantanal (Costa 1999). Já no século XX, Frederico Carlos Hoehne participou de expedições ao Mato Grosso onde coletou e identificou centenas de espécies (Franco \& Drummond 2005) e foi provavelmente o primeiro pesquisador brasileiro que listou espécies alimentícias nativas (Hoehne 1946) do estado de "Mato Grosso" (atualmente dividido em Mato Grosso e Mato Grosso do Sul). Espécies alimentícias nativas e usos conhecidos no Brasil até meados do século passado foram registrados por Pio Corrêa (1926-1978), também com citação das espécies para o então estado de Mato Grosso.

Os trabalhos de Conceição \& Paula (1986), Berg (1986), Conceição \& Paula (1990), Pott \& Pott (1994), Pott \& Pott (2000b) e Pott et al. (2004) são as publicações mais recentes sobre plantas do Pantanal, que incluem as alimentícias, já com indicação de ocorrência para o atual estado de Mato Grosso do Sul. Além desses, Oliveira (1996) mencionou 18 espécies nativas com uso alimentício pelos índios Guató, habitantes do Pantanal. Damasceno-Junior et al. (2010) foi o primeiro trabalho voltado especificamente para plantas alimentícias no estado, associando informações sobre o valor nutricional e indicação de uso na culinária, com identificação das espécies baseadas em coletas botânicas e depósito em Herbário. O trabalho de Bortolotto et al. (2015) 
contém informações sobre plantas alimentícias usadas ou conhecidas por moradores de comunidades rurais do Pantanal.

A falta de uma listagem atual das espécies alimentícias do Mato Grosso do Sul, com identificação taxonômica realizada por especialistas (com base em material botânico depositado em Herbário), limita os estudos relacionados ao uso, manejo e conservação das espécies nativas, bem como aqueles voltados ao conhecimento sobre o valor nutricional, à tecnologia de alimentos e à segurança alimentar. Uma lista de espécies alimentícias do estado com dados sobre as fitofisionomias onde são encontradas é importante também para subsidiar políticas públicas relacionadas ao uso e conservação da flora. Este trabalho tem o objetivo de organizar uma lista das espécies alimentícias nativas do Mato Grosso do Sul ou potencialmente úteis para a dieta humana, e destacar as fisionomias vegetais do estado onde essas espécies ocorrem.

Não há uma listagem de todas as plantas alimentícias do mundo (Kinupp \& Barros 2004). Esses autores citaram a ocorrência de 2000 espécies não convencionais para o Brasil e mencionaram como um dos trabalhos mais completos sobre plantas alimentícias ou potencialmente alimentícias no mundo o trabalho de Kunkel (1984) onde são citadas 12.500 espécies.

Os principais grupos de pesquisas sobre plantas alimentícias no Mato Grosso do Sul estão relacionados ao programa de Pós-Graduação em Biologia Vegetal e ao Programa de Pós Graduação em Saúde e Desenvolvimento na Região Centro-Oeste da Universidade Federal de Mato Grosso do Sul (Grupo de estudos da flora e tecnologia de alimentos, respectivamente) e na Universidade Federal da Grande Dourados. Nas duas instituições os grupos de pesquisas se dedicam a estudos florísticos e etnobotânicos e têm parcerias com grupos de estudos em tecnologia em alimentos e de germinação. Essas parcerias têm sido fundamentais para o desenvolvimento de projetos de pesquisa e extensão interdisciplinares relacionados à diversidade de plantas alimentícias.

O Mato Grosso do Sul tem ainda poucos estudos etnobotânicos com registros na literatura sobre formas de uso e manejo da flora, feitos tradicionalmente pelas populações humanas para fins alimentícios e poucos profissionais nas instituições de ensino e pesquisa capacitados para atuar nessa área. Paradoxalmente, é o segundo estado brasileiro com a maior população indígena do país, ocupando áreas com fitofisionomias nativas ainda pouco estudadas.

Estudos futuros que busquem identificar usos de espécies podem dar subsídios aos usos de plantas não convencionais, bem como nomes locais usados pelas populações indígenas. Outros estudos que incluam consultas a documentos históricos ou uma revisão de estudos etnológicos também são importantes.

Estudos relacionados às fisionomias vegetais do estado onde ocorrem espécies alimentícias também são ainda restritos. Merecem destaque o Chaco e as Veredas. Ambos são bem representados no Mato Grosso do Sul e pouco estudados. A responsabilidade relacionada aos estudos no Chaco aumenta quando consideramos que este é o único estado brasileiro com essa fisionomia vegetal, ainda com poucas áreas protegidas e com riscos relacionados a desmatamentos.

\section{MATERIAL E MÉTODOS}

Incluímos nesta listagem espécies alimentícias para o homem, nativas do Mato Grosso do Sul, compiladas de fontes bibliográficas, cujas coletas foram feitas no estado e com identificação baseadas em material testemunho com depósito em herbário.Diversos números de coletor mencionados nas publicações foram mantidos e citados neste trabalho.

Foram incluídas espécies alimentícias coletadas pelos autores no período de 1980 a 2012 nas áreas com vegetação nativa no Mato Grosso do Sul e depositadas nos três principais herbários do estado: CGMS, COR e CPAP. As informações sobre o uso alimentício dessas espécies foram obtidas na literatura (mencionada para o Mato Grosso do Sul, para outros estados brasileiros, ou para os países vizinhos: Bolívia, Argentina e Paraguai, que compartilham as fisionomias nativas); por degustação (feita pelos autores no campo, durante a coleta botânica) e pela observação de espécies comercializadas em feiras, nas ruas ou em estradas. Algumas espécies da família Arecaceae e dos gêneros Dioscorea e Passiflora, que integram o banco de dados online de taxonomia/florística citadas para o Mato Grosso do Sul na Lista de Espécies da Flora do Brasil (2012), foram incluídas neste trabalho, somadas às coletadas pelos autores. O número do coletor para essas espécies, sem registros para os herbários COR, CPAP e CGMS, foram obtidos da Lista de Espécies da Flora do Brasil (2013), quando mencionada para o Mato Grosso do Sul ou no Herbário Virtual da Flora e dos Fungos (INCT 2012).

A lista apresentada neste trabalho consta de espécies usadas ou com potencial de uso para consumo in natura, para preparo de farinhas (crua ou torrada), sal, bebidas, usadas em temperos, preparadas cozidas (doces, por exemplo) e outros. Foram incluídas espécies usadas na forma de chá somente como uma bebida saborosa ou para consumo durante ou após a refeição, mas não usadas para tratar da saúde como um chá medicinal. As informações sobre as fisionomias vegetais foram obtidas das etiquetas (ou caderneta de campo dos autores). O número de fitofisionomias onde a espécie ocorre foi usado para estimar quais são as mais ricas em número de espécies alimentícias no estado.

O termo plantas nativas foi utilizado para se referir às espécies que são encontradas na flora nativa do estado. Algumas dessas espécies, de ampla distribuição, que são também encontradas próximas a ambientes domésticos (sedes de fazendas, sítios e outros) como a Acrocomia aculeata, por exemplo, consideradas subespontâneas, foram incluídas e há um destaque diferenciando-as. Os nomes 
populares foram mantidos apenas quando foi possível identificar que se tratava de nomes locais, usados no Mato Grosso do Sul. Os nomes da etnia Guató mencionados por Oliveira (1996) ou Guarani como mencionados por Lorenzi et al. (2010) para as plantas alimentícias foram inseridos com os nomes locais, assim como nomes indígenas encontrados em trabalhos sobre histórica como em Herberts (1998).

\section{RESULTADOS E DISCUSSÃO}

Foram listadas 293 espécies de plantas alimentícias para o Mato Grosso do Sul das quais 141 não tinham citação sobre o potencial alimentício na literatura para o estado. As principais fontes com informações sobre as espécies alimentícias consultadas estão apresentadas na Tabela 1. Outras informações sobre as espécies compiladas constam principalmente dos trabalhos de Berg (1986), Conceição \& Paula (1986 e 1990) e Oliveira (1996). Não houve acréscimos às espécies alimentícias aquáticas apresentadas neste trabalho em relação às apresentadas Pott \& Pott (2000b).

Tabela 1. Fontes bibliográficas que citam o uso alimentício das espécies nativas do Mato Grosso do Sul, com material depositado em Herbário.

\begin{tabular}{lc}
\hline Referência & Número de espécies \\
\hline Pott \& Pott (1994) & 104 \\
Pott et al. (2004) & 99 \\
Damasceno-Junior et al.(2010) & 60 \\
Pott \& Pott (2000b) & 21 \\
Bortolotto et al. (2015) & 54 \\
\hline
\end{tabular}

Os nomes populares levantados (Quadro 1) refletem a diversidade cultural do estado de Mato Grosso do Sul como consequência da situação fronteiriça com o Paraguai e Bolívia. As espécies sem nome local refletem a necessidades de estudos etnobotânicos relacionados às plantas conhecidas pelas populações humanas tais como indígenas, tradicionais, quilombolas ou de pequenos agricultores do Mato Grosso do Sul. As espécies encontradas estão distribuídas em 160 gêneros e 67 famílias botânicas, sendo as mais numerosas apresentadas na Figura 1. Dentre as Fabaceae, destacamos espécies frutíferas, com grande valor nutricional e crescente valor econômico no Mato Grosso do Sul e no Brasil, como Dipteryx alata, e Hymenaea spp., que atualmente vêm sendo comercializadas no MS por pequenos agricultores. Merecem destaque também os ingás (Inga spp.), que têm sido utilizados por seus frutos comestíveis por milhares de anos e hoje ainda constituem um item importante do comércio local em países andinos, América Central e do Sul (Pennington 1997). A família Myrtaceae é amplamente disseminada por todas as regiões tropicais do mundo (Judd et al.1999).

Há várias espécies pertencentes às famílias e gêneros mais numerosos que não têm comprovação de uso, como as pertencentes aos gêneros Arachis (30 espécies), Eugenia

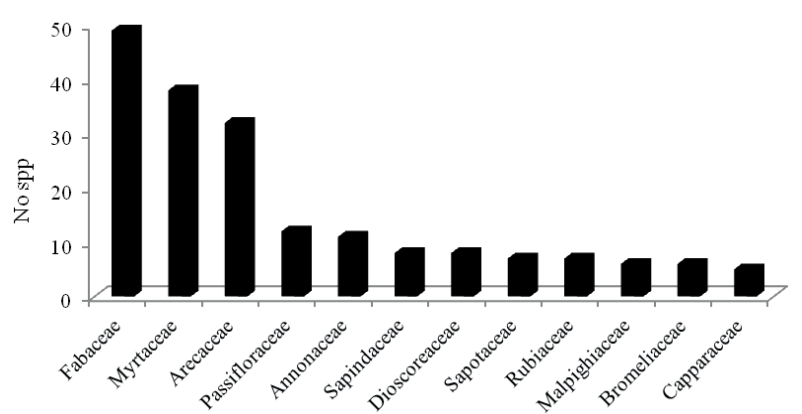

Fig. 1. Famílias botânicas com maior número de espécies alimentícias (ou com potencial alimentício) no estado de Mato Grosso do Sul.

(17), Passiflora (12), Syagrus (nove) e Butia (seis), dentre outras (Quadro 1). As espécies sem informação de uso no Quadro 1 estão listadas aqui para chamar a atenção para a necessidade de estudos científicos voltados à Etnobotânica, Biologia, valor nutricional e conservação. Além disso, a inclusão de Arachis com todas as espécies citadas por Valls (2012) foi feita, ainda que muitas delas tenham sementes diminutas, produzam pouco e não tenham comprovação de uso na dieta humana, uma vez que espécies silvestres têm sido utilizadas no melhoramento do Arachis hypogaea (Valls 2005). Eugenia e Passiflora incluem espécies com frutos geralmente comestíveis. Os maracujás (Passiflora spp.) têm arilos comestíveis, amplamente utilizados na dieta humana. Hoehne (1946) menciona cerca de 150 espécies nativas, das quais "mais de 60 podem ser aproveitadas como alimento". Entretanto, há relatos de espécies do gênero com toxicidade (Armando C. Cervi com. pess).

A família Arecaceae tem potencial para aproveitamento dos frutos frescos, na forma de farinhas (da polpa e endosperma), bebidas frescas ou alcoólicas, óleos comestíveis ou palmito e também inclui espécies sem indicação de uso. Do "butiazinho azedo" (Butia matogrossensis), por exemplo, não há informação sobre o uso alimentício na literatura (Lorenzi et al. 2010), mas seu nome popular sugere o sabor do fruto, merecendo interesse por estudos sobre seu valor nutricional e potencial alimentício.

Além das espécies com usos ainda pouco conhecidos, há aquelas com comprovado valor nutricional como Acrocomia aculeata, por exemplo. Essa espécie tem o mesocarpo rico em carotenóides (Ramos et al. 2008) e é usado para produção de farinha, sorvetes ou licores em Corumbá (Pott \& Pott 1994). A única espécie usada para palmito que tem cultivo para fins alimentícios é a guariroba (Syagrus oleracea), com o palmito de sabor amargo comercializado em feiras na capital (Campo Grande) e o produto congelado ou na forma de conserva já comercializado por pequenas empresas (Soares 2009). O gênero Syagrus, que ocorre com nove espécies neste trabalho (Quadro 1), inclui palmeiras comumente utilizadas para aproveitamento do palmito na América do Sul, juntamente com Geonoma (Haynes \& McLaughlin 2000). 
Quadro 1. Espécies alimentícias do Mato Grosso do Sul - Brasil com nome popular usado no Mato Grosso do Sul*, Nome indígena Guató (Gt), Guarani (Gr) e Mbayá-Guaicurú (Mb), nome e número do coletor, herbário e fisionomia vegetal onde ocorre: Mata Ribeirinha (MR), Chaco (CH), Cerrado latu sensu (CE), Floresta Estacional Decidual (FED), Floresta Estacional Semidecidual (FES), Campo Inundável no Pantanal (CIP), Campo Inundável - fora do Pantanal/inclui veredas (CI), Ruderal (RU), Cultivada (CUL). Fonte da informação: 0 = Sem informação de uso. Uso potencial: 1 = Pott \& Pott (1994); 2 = Pott et al. (2004); 3 = Damasceno-Junior et al. (2010); $4=$ Pott \& Pott (2000 b); $5=$ Bortolotto et al. (2015); $6=$ uso observado pelos autores ou que consta na etiqueta do herbário; 7 = Lorenzi et al. (2006); $8=$ Lorenzi et al. (2010); $9=$ Soares 2009 (10) Hoehne (1946); 11 UMSA et al. (2002); 12=Amaral \& Guarim Neto (2008); $13=$ Kinupp (2007); $14=$ Arenas \& Scarpa (2007); 15 = Pio Corrêa (1926-1978); 16 = Haynes \& Mclaughlin (2000); $17=$ Lewis (1992); 18 = Mostacedo \& Uslar 1999; $19=$ Susnik (1982); $20=$ Pedralli (2002); 21 = Ministério da Saúde (2002); 22 = Bezerra et al. (2006); 23 = Martins et al. (2003), 24 = Oliveira (1996), 25= Conceição \& Paula (1990); 26 = Valls (2012), $27=$ Herberts (1998).

\begin{tabular}{|c|c|c|c|c|c|c|}
\hline Família & Nome científico & $\begin{array}{l}\text { Nome popular no MS* } \\
\text { "nome indígena"e (fonte) }\end{array}$ & $\begin{array}{l}\text { Nome e Número } \\
\text { do coletor }\end{array}$ & Herbário & Fisionomia & Fontes \\
\hline Alismataceae & $\begin{array}{l}\text { Echinodorus grandiflorus } \\
\text { (Cham. \& Schltr.) Micheli }\end{array}$ & Chapéu-de-couro* & V. J. Pott 4952 & CPAP & CIP & 4 \\
\hline Alismataceae & Limnocharis flava (L.) Buchenau & Camalote* & A. Pott 4736 & CPAP & CIP & 4 \\
\hline Alismataceae & $\begin{array}{l}\text { Lophiocarpus guayanensis } \\
\text { (Kunth) Micheli }\end{array}$ & - & V. J. Pott 2649 & CPAP & CIP & 4 \\
\hline Amaranthaceae & Amaranthus spinosus L. & Caruru-chifre-de-espinho* & $\begin{array}{l}\text { G. A. Damasceno- } \\
\text { Junior } 4725\end{array}$ & COR & RU & 2 \\
\hline Amaranthaceae & Amaranthus viridis L. & Caruru* & $\begin{array}{l}\text { I. M. Bortolotto } \\
1063\end{array}$ & COR & CIP, RU & 1,5 \\
\hline Anacardiaceae & Anacardium humile A.St.-Hil. & Cajuzinho* & A. Pott 4380 & CPAP & $\mathrm{CE}$ & $\begin{array}{c}1,2,3 \\
6,7\end{array}$ \\
\hline Anacardiaceae & Schinus terebinthifolius Raddi & $\begin{array}{l}\text { Pimenta-do-reino-de- } \\
\text { árvore* }\end{array}$ & $\begin{array}{l}\text { C.A. Conceição } \\
1832\end{array}$ & CGMS & $\mathrm{MR}, \mathrm{CE}$, & 9 \\
\hline Anacardiaceae & Spondias mombin L. & Caiá*, acaiá* & $\begin{array}{l}\text { G. A. Damasceno- } \\
\text { Junior } 3119\end{array}$ & COR & $\begin{array}{l}\text { MR, FES, } \\
\text { RU }\end{array}$ & 3,5 \\
\hline Annonaceae & Annona cacans Warm. & - & U. Pastore116 & MBM & $\mathrm{CE}$ & 10 \\
\hline Annonaceae & Annona coriacea Mart. & $\begin{array}{l}\text { Araticum*, marolo*, } \\
\text { pinha-do-cerrado* }\end{array}$ & $\begin{array}{l}\text { G. A. Damasceno- } \\
\text { Junior } 3811\end{array}$ & COR & $\mathrm{CE}$ & $3,6,7$ \\
\hline Annonaceae & Annona cornifolia A. St.-Hil. & Ata-de-cobra* & $\begin{array}{l}\text { I. M. Bortolotto } \\
1234\end{array}$ & COR & $\mathrm{CE}$ & $1,2,5$ \\
\hline Annonaceae & Annona crassiflora Mart. & $\begin{array}{c}\text { Araticum*, araticum-da- } \\
\text { mata* }\end{array}$ & $\begin{array}{l}\text { G. A. Damasceno- } \\
\text { Junior } 4439\end{array}$ & COR & $\mathrm{CE}$ & 7 \\
\hline Annonaceae & Annona dioica A. St.-Hil. & - & A. Pott 4467 & CPAP & $\mathrm{CE}$ & $1,2,6$ \\
\hline Annonaceae & $\begin{array}{c}\text { Annona emarginata (Schltdl.) } \\
\text { H. Rainer }\end{array}$ & Arixicum-do-mato* & $\begin{array}{l}\text { G. A. Damasceno- } \\
\text { Junior } 5012\end{array}$ & COR & $\begin{array}{l}\text { CH, FED, } \\
\text { FES }\end{array}$ & 1,2 \\
\hline Annonaceae & Annona montana Macfad. & Ata-brava* & $\begin{array}{l}\text { G. A. Damasceno- } \\
\text { Junior } 2843\end{array}$ & COR & $\begin{array}{l}\text { MR, FES, } \\
\text { RU }\end{array}$ & 6,7 \\
\hline Annonaceae & $\begin{array}{c}\text { Annona nutans (R.E. Fr. ) R.E. } \\
\text { Fr. }\end{array}$ & Ata* & $\begin{array}{l}\text { G. A. Damasceno- } \\
\text { Junior } 2459\end{array}$ & COR & FED & 5,11 \\
\hline Annonaceae & $\begin{array}{l}\text { Duguetia furfuracea (A.St.-Hil.) } \\
\text { Saff. }\end{array}$ & Ariticunzinho* & $\begin{array}{l}\text { G. A. Damasceno- } \\
\text { Junior } 4378\end{array}$ & COR & $\mathrm{CE}$ & 1,2 \\
\hline Annonaceae & $\begin{array}{c}\text { Rollinia sylvatica (A. St.-Hil.) } \\
\text { Mart. }\end{array}$ & $\begin{array}{l}\text { Araticum-do-mato, } \\
\text { araticum-da-mata }\end{array}$ & $\begin{array}{l}\text { G. A. Damasceno- } \\
\text { Junior } 3132\end{array}$ & COR & FES & 0 \\
\hline Annonaceae & Xylopia aromatica (Lam.) Mart. & $\begin{array}{l}\text { Pindaíva*, pindaíba*, } \\
\text { pimenta-de-macaco* }\end{array}$ & $\begin{array}{l}\text { G. A. Damasceno- } \\
\text { Junior } 2324\end{array}$ & COR & $\mathrm{CE}$ & 1,3 \\
\hline Apiaceae & $\begin{array}{l}\text { Eryngium pandanifolium Cham. } \\
\text { \& Schltdl. }\end{array}$ & - & $\begin{array}{l}\text { G. A. Damasceno- } \\
\text { Junior } 3833\end{array}$ & COR & $\mathrm{CI}$ & 13 \\
\hline Apiaceae & $\begin{array}{l}\text { Eryngium elegans Cham. \& } \\
\text { Schltdl. }\end{array}$ & - & A. Pott 3580 & CPAP & CIP, CI, & 13 \\
\hline Apocynaceae & $\begin{array}{l}\text { Aspidosperma quebracho-blanco } \\
\text { Schltdl. }\end{array}$ & $\begin{array}{l}\text { Quina*, quina-da- } \\
\text { morraria* }\end{array}$ & $\begin{array}{l}\text { T.S.Conceição } \\
\text { (16) }\end{array}$ & COR & CH, FED, & 14 \\
\hline Apocynaceae & Hancornia speciosa Gomez & Mangaba*, mangava* & I. M. Bortolotto 1211 & COR & $\mathrm{CE}$ & $\begin{array}{l}1,2 \\
3,5\end{array}$ \\
\hline Aquifoliaceae & Ilex paraguariensis A. St.-Hil. & Erva-mate* & U.M. Resende 1243 & CGMS & FES & 5 \\
\hline Araceae & Pistia stratiotes L. & Alface-d'água & V. J. Pott 2918 & CPAP & CIP, & 4 \\
\hline Araceae & $\begin{array}{l}\text { Urospatha sagittifolia (Rudge) } \\
\text { Schott }\end{array}$ & - & V. J. Pott 2679 & CPAP & CI, CIP, & 4 \\
\hline Araceae & $\begin{array}{l}\text { Xanthosoma riedelianum } \\
\text { (Schott) Schott }\end{array}$ & - & V. J. Pott 3043 & CPAP & CIP, CI, & 4 \\
\hline Arecaceae & $\begin{array}{c}\text { Acrocomia aculeata (Jacq.) } \\
\text { Lodd. ex Mart. }\end{array}$ & $\begin{array}{c}\text { Bocaiuva*, 'Namogoligi”' } \\
\text { (Mb, 27), "Maguedji”' (Gt, 24) }\end{array}$ & V. J. Pott 2408 & CPAP & $\begin{array}{l}\text { CE, FES, } \\
\text { RU }\end{array}$ & $\begin{array}{l}1,2,3 \\
5,7,8 \\
24,27\end{array}$ \\
\hline
\end{tabular}


Quadro 1. Cont.

\begin{tabular}{|c|c|c|c|c|c|c|}
\hline Família & Nome científico & $\begin{array}{l}\text { Nome popular no MS* } \\
\text { “nome indígena"e (fonte) }\end{array}$ & $\begin{array}{l}\text { Nome e Número } \\
\text { do coletor }\end{array}$ & Herbário & Fisionomia & Fontes \\
\hline Arecaceae & $\begin{array}{c}\text { Allagoptera leucocalyx (Drude) } \\
\text { Kuntze }\end{array}$ & Buri* & A. Pott 4306 & CPAP & $\mathrm{CE}$ & $\begin{array}{l}1,2,5 \\
10\end{array}$ \\
\hline Arecaceae & $\begin{array}{c}\text { Allagoptera campestris (Mart.) } \\
\text { Kuntze }\end{array}$ & - & $\begin{array}{l}\text { H. Lorenzi, R. } \\
\text { Pimenta \& R. } \\
\text { Campos } 6689\end{array}$ & HPL & $\mathrm{CE}$ & 0 \\
\hline Arecaceae & Attalea geraensis Barb. Rodr. & Pindó*, inajá* & A. Pott 11605 & CGMS & CE, FES & 6 \\
\hline Arecaceae & $\begin{array}{c}\text { Attalea phalerata (Mart.) ex } \\
\text { Spreng. }\end{array}$ & $\begin{array}{l}\text { Acuri*, bacuri*, "mudjí “ } \\
(\mathrm{Gt}, 24)\end{array}$ & A. Pott 4427 & CPAP & $\begin{array}{l}\mathrm{MR}, \mathrm{CE} \\
\mathrm{FES}\end{array}$ & $\begin{array}{l}1,2,3 \\
5,7,8\end{array}$ \\
\hline Arecaceae & $\begin{array}{c}\text { Attalea speciosa Mart. ex } \\
\text { Spreng. }\end{array}$ & Babaçu*, aguaçu ( 1) & A. Pott 6454 & CPAP & MR, FES & 1,2 \\
\hline Arecaceae & Bactris glaucescens Drude & $\begin{array}{l}\text { Tucum*, tucum-azedo*, } \\
\text { tucum-preto*, tucum- } \\
\text { roxo*, "magueto" }(\mathrm{Gt}, 24)\end{array}$ & $\begin{array}{l}\text { I. M. Bortolotto } 1339 \text {, } \\
\text { G. A. Damasceno- } \\
\text { Junior } 305\end{array}$ & COR & MR & $\begin{array}{l}1,2,3 \\
5,7,8 \\
24\end{array}$ \\
\hline Arecaceae & Bactris major Jacq. & $\begin{array}{l}\text { Tucum-branco*, tucum- } \\
\text { doce* e tucum ouriço* }\end{array}$ & $\begin{array}{l}\text { I. M. Bortolotto } \\
973\end{array}$ & COR & MR & 5,8 \\
\hline Arecaceae & Bactris riparia Mart. & $\begin{array}{l}\text { Tucum-vermelho*, tucum- } \\
\text { guaçu* }\end{array}$ & $\begin{array}{l}\text { G. A. Damasceno- } \\
\text { Junior } 2344\end{array}$ & COR & MR & 5,8 \\
\hline Arecaceae & $\begin{array}{c}\text { Butia campicola (Barb. Rodr.) } \\
\text { Noblick }\end{array}$ & "Yataicapii” (Gr, 8) & $\begin{array}{l}\text { H. Lorenzi, K. Soares } \\
\text { \& R. Campos } 6771\end{array}$ & HPL & $\mathrm{CE}$ & 0 \\
\hline Arecaceae & Butia exospadix Noblick & “Jataí-poñy”(Gr, 8) & $\begin{array}{l}\text { H. Lorenzi, K. Soares } \\
\text { \& R. Campos } 6772\end{array}$ & HPL & FES & 0 \\
\hline Arecaceae & Butia lepidotispatha Noblick. & Butiá-azul-do-cerrado* (8 & $\begin{array}{l}\text { Lorenzi, H. } 6767 \\
\text { HP (citado por } \\
\text { Lorenzi 2010) }\end{array}$ & HPL & $\mathrm{CE}$ & 0 \\
\hline Arecaceae & $\begin{array}{c}\text { Butia leptospatha (Burret) } \\
\text { Noblick }\end{array}$ & - & $\begin{array}{l}\text { H. Lorenzi, K. Soares } \\
\text { \& R. Campos } 6769\end{array}$ & HPL & $\mathrm{CE}$ & 15 \\
\hline Arecaceae & $\begin{array}{c}\text { Butia paraguayensis (Barb. } \\
\text { Rodr.) Bailey }\end{array}$ & Butiá*, cabeçudo* & U.M. Resende 229 & CGMS & $\mathrm{CE}$ & 3,8 \\
\hline Arecaceae & $\begin{array}{c}\text { Butia matogrossensis Noblick \& } \\
\text { Lorenzi }\end{array}$ & Butiazinho-azedo* (8) & R. Tsuji 2378 & HPL & $\mathrm{CE}$ & 0 \\
\hline Arecaceae & $\begin{array}{c}\text { Copernicia alba Morong ex } \\
\text { Morong \& Britton }\end{array}$ & Carandá*, "mufá” $(\mathrm{Gt}, 24)$ & A. Pott 5430 & CPAP & $\mathrm{CH}, \mathrm{CIP}$ & $1,5,24$ \\
\hline Arecaceae & Desmoncus orthacanthos Mart. & Urubamba* & $\begin{array}{l}\text { I. M. Bortolotto } \\
1281\end{array}$ & COR & MR & 5 \\
\hline Arecaceae & Desmoncus polyacanthos Mart. & Urubamba* & $\begin{array}{l}\text { Registro visual: } \\
\text { Geraldo Alves } \\
\text { Damasceno Junior }\end{array}$ & $\begin{array}{l}\text { Sem } \\
\text { registro }\end{array}$ & $\mathrm{CE}$ & 6 \\
\hline Arecaceae & Euterpe edulis Mart. & Palmito & V. J. Pott 7385 & CGMS & CE, FES & 6,8 \\
\hline Arecaceae & Euterpe precatoria Mart. & Açaí & $\begin{array}{c}\text { Registro } \\
\text { visual:Geraldo } \\
\text { Alves Damasceno } \\
\text { Junior }\end{array}$ & $\begin{array}{l}\text { Sem } \\
\text { registro }\end{array}$ & $\mathrm{CE}$ & 6,8 \\
\hline Arecaceae & $\begin{array}{c}\text { Geonoma brevispatha Barb. } \\
\text { Rodr. }\end{array}$ & - & $\begin{array}{l}\text { U.M. Resende } \\
1153\end{array}$ & CGMS & $\mathrm{CE}$ & 0 \\
\hline Arecaceae & Mauritia flexuosa L. f. & Buriti* & A. Pott 5030 & CPAP & CIP, CI & $1,2,3,8$ \\
\hline Arecaceae & $\begin{array}{l}\text { Syagrus campylospatha (Barb. } \\
\text { Rodr.) Becc. }\end{array}$ & $\begin{array}{l}\text { "Yatai-mi”, "yatay- } \\
\text { pequeño" (8) }\end{array}$ & $\begin{array}{l}\text { H. Lorenzi, R. } \\
\text { Pimenta \& R. } \\
\text { Campos } 6694\end{array}$ & HPL & $\mathrm{CI}$ & 0 \\
\hline Arecaceae & $\begin{array}{c}\text { Syagrus cerqueirana Noblick \& } \\
\text { Lorenzi }\end{array}$ & Acumã-mirim* (8) & H. Lorenzi 6578 & HPL & $\mathrm{CE}$ & 0 \\
\hline Arecaceae & Syagrus comosa (Mart.) Mart. & Palmito amargo* & H. Lorenzi 6579 & HPL & $\mathrm{CE}$ & 8 \\
\hline Arecaceae & Syagrus flexuosa (Mart.) Becc. & Acumã, acumãn (1) & A. Pott 4389 & CPAP & $\mathrm{CE}$ & $\begin{array}{l}1,2,7 \\
8,10\end{array}$ \\
\hline Arecaceae & $\begin{array}{l}\text { Syagrus graminifolia (Mart.) } \\
\text { Becc. }\end{array}$ & Palmeirinha (8) & H. Lorenzi 2805 & HPL & $\begin{array}{c}\mathrm{CE} \\
\mathrm{MR} \text { FES }\end{array}$ & 0 \\
\hline Arecaceae & Syagrus oleracea (Mart.) Becc. & Guariroba* & U.M. Resende 722 & CGMS & $\begin{array}{l}\text { MR, FES, } \\
\text { CUL }\end{array}$ & $7,8,9$ \\
\hline Arecaceae & Syagrus petraea (Mart.) Becc. & - & U.M. Resende 478 & CGMS & $\mathrm{CE}$ & 0 \\
\hline Arecaceae & $\begin{array}{c}\text { Syagrus procumbens Noblick \& } \\
\text { Lorenzi }\end{array}$ & Ariri rasteiro (8) & $\begin{array}{l}\text { H. Lorenzi, K. } \\
\text { Soares \& R. } \\
\text { Campos } 6779\end{array}$ & HPL & $\mathrm{CE}$ & 0 \\
\hline
\end{tabular}


Quadro 1. Cont.

\begin{tabular}{|c|c|c|c|c|c|c|}
\hline Família & Nome científico & $\begin{array}{l}\text { Nome popular no MS* } \\
\text { "nome indígena"e (fonte) }\end{array}$ & $\begin{array}{l}\text { Nome e Número } \\
\text { do coletor }\end{array}$ & Herbário & Fisionomia & Fontes \\
\hline Arecaceae & $\begin{array}{c}\text { Syagrus romanzoffiana (Cham.) } \\
\text { Glassman }\end{array}$ & Pindó*, jerivá* & $\begin{array}{l}\text { G. A. Damasceno- } \\
\text { Junior } 2106\end{array}$ & CGMS & FES & 3,8 \\
\hline Arecaceae & Trithrinax schizophylla Drude & Carandilla, carandaí (8) & $\begin{array}{l}\text { Registro visual } \\
\text { - Geraldo alves } \\
\text { Damasceno Junior. } \\
\text { Citada por Lorenzi } \\
\text { (2010) para o MS. }\end{array}$ & VER & $\mathrm{CH}$ & 16 \\
\hline Asteraceae & Pacourina edulis Aubl. & - & V. J. Pott 2520 & CPAP & CIP & 4 \\
\hline Asteraceae & $\begin{array}{c}\text { Stevia rebaudiana (Bertoni) } \\
\text { Bertoni }\end{array}$ & Caá-êhê (Gr, 17) & $\begin{array}{l}\text { I. M. Bortolotto } \\
215\end{array}$ & COR & $\mathrm{CE}$ & 17 \\
\hline Bignoniaceae & $\begin{array}{l}\text { Tabebuia aurea (Silva Manso) } \\
\text { Benth. \& Hook. f. ex S. Moore }\end{array}$ & Paratudo* & A. Pott 3281 & CPAP & $\begin{array}{l}\text { MR, CH, } \\
\text { CE, CIP }\end{array}$ & 1,2 \\
\hline Bignoniaceae & $\begin{array}{c}\text { Tabebuia heptaphylla (Vell.) } \\
\text { Toledo }\end{array}$ & $\begin{array}{c}\text { Piúva*, piúva-do- } \\
\text { Pantanal*, piúva-roxa*, } \\
\text { piúva-do-campo*, peúva* }\end{array}$ & $\begin{array}{l}\text { G. A. Damasceno- } \\
\text { Junior } 391\end{array}$ & $\mathrm{COR}$ & MR & 1 \\
\hline Bromeliaceae & Aechmea distichantha Lem. & Caraguatá-chuçá* & AP 4412 & CPAP & CE, FES & 1 \\
\hline Bromeliaceae & $\begin{array}{l}\text { Ananas ananassoides (Baker) } \\
\text { L. B. Sm. }\end{array}$ & $\begin{array}{c}\text { Abacaxizinho do cerrado, } \\
\text { abacaxizinho }\end{array}$ & A. Pott 4382 & CPAP & $\mathrm{CE}$ & $\begin{array}{l}1,2 \\
3,5\end{array}$ \\
\hline Bromeliaceae & Bromelia balansae $\mathrm{Mez}$ & Caraguatá*, gravatá* & V. J. Pott 714 & CPAP & $\begin{array}{l}\mathrm{MR}, \mathrm{CH} \\
\mathrm{CE}, \mathrm{FED}, \\
\text { FES }\end{array}$ & $1,2,3$ \\
\hline Bromeliaceae & Bromelia interior L.B.Sm. & - & P. I. Oliveira 10 & MBM & $\mathrm{CE}$ & 6 \\
\hline Bromeliaceae & Bromelia serra Griseb. & - & $\begin{array}{c}\text { G. Hatschsbach } \\
58809\end{array}$ & MBM & $\mathrm{CE}$ & 14 \\
\hline Bromeliaceae & $\begin{array}{c}\text { Pseudananas } \\
\text { sagenarius (Arruda) Camargo }\end{array}$ & Abacaxi-do-mato & AP 12350 & CGMS & FES & 6 \\
\hline Burseraceae & $\begin{array}{c}\text { Protium heptaphyllum (Aubl.) } \\
\text { Marchand }\end{array}$ & $\begin{array}{l}\text { Almécega*, almésca*, } \\
\text { armésca*, amécicla (1) }\end{array}$ & $\begin{array}{l}\text { G. A. Damasceno- } \\
\text { Junior } 1256\end{array}$ & $\mathrm{COR}$ & MR, FES & 1,2 \\
\hline Cactaceae & $\begin{array}{l}\text { Brasiliopuntia brasiliensis } \\
\text { (Willd.) A. Berger }\end{array}$ & - & $\begin{array}{l}\text { G. A. Damasceno- } \\
\text { Junior } 2668\end{array}$ & COR & MR, FES & 18 \\
\hline Cactaceae & $\begin{array}{c}\text { Cereus bicolor Rizzini \& A. } \\
\text { Mattos }\end{array}$ & Urumbeva* & $\begin{array}{l}\text { G. A. Damasceno- } \\
\text { Junior } 1014\end{array}$ & COR & FED, FES & 5 \\
\hline Cactaceae & $\begin{array}{c}\text { Epiphyllum phyllanthus (L.) } \\
\text { Hawk. }\end{array}$ & - & A. Pott 11749 & CGMS & MR & 6 \\
\hline Cactaceae & Pereskia sacharosa Griseb. & - & $\begin{array}{l}\text { G. A. Damasceno- } \\
\text { Junior } 2009\end{array}$ & COR & CH, FED & 1,2 \\
\hline Calophyllaceae & $\begin{array}{c}\text { Calophyllum brasiliense } \\
\text { Cambess. }\end{array}$ & Guanandi* & $\begin{array}{l}\text { G. A. Damasceno- } \\
\text { Junior } 4412\end{array}$ & COR & MR, FES & 1,2 \\
\hline Cannabaceae & $\begin{array}{l}\text { Celtis pubescens (Kunth) } \\
\text { Spreng. }\end{array}$ & Taleira* & A. Pott 3787 & CPAP & $\begin{array}{l}\text { MR, } \mathrm{CH} \\
\text { CE, FED }\end{array}$ & $1,2,11$ \\
\hline Cannabaceae & Celtis spinosa Spreng. & - & A. Pott 5287 & CPAP & $\begin{array}{l}\text { MR, CE, } \\
\text { FES }\end{array}$ & 1,2 . \\
\hline Cannaceae & Canna glauca $\mathrm{L}$. & $\begin{array}{l}\text { Cana-do-brejo*, cana*, } \\
\text { bananinha-do-brejo* }\end{array}$ & V. J. Pott 2150 & CPAP & CIP, CI & 2,4 \\
\hline Capparaceae & $\begin{array}{c}\text { Anisocapparis speciosa (Griseb.) } \\
\text { Cornejo \& Iltis }\end{array}$ & $\begin{array}{l}\text { Mangaba-brava*, } \\
\text { mangava-brava* }\end{array}$ & $\begin{array}{l}\text { G. A. Damasceno- } \\
\text { Junior } 2699\end{array}$ & COR & FED & 2,3 \\
\hline Capparaceae & $\begin{array}{l}\text { Capparicordis tweediana } \\
\text { (Eichler) Iltis \& Cornejo }\end{array}$ & - & $\begin{array}{l}\text { G. A. Damasceno- } \\
\text { Junior } 1928\end{array}$ & COR & $\mathrm{CH}, \mathrm{FED}$ & 14 \\
\hline Capparaceae & $\begin{array}{l}\text { Capparidastrum petiolare } \\
\text { (Kunth) Hutch. }\end{array}$ & - & $\begin{array}{l}\text { G. A. Damasceno- } \\
\text { Junior } 2667\end{array}$ & COR & FED, FES & 6 \\
\hline Capparaceae & Crataeva tapia $\mathrm{L}$. & $\begin{array}{l}\text { Cabaça*, cabaceira-do- } \\
\text { Pantanal, cabeceira (1) }\end{array}$ & A. Pott 6971 & CPAP & MR & $1,2,7$ \\
\hline Capparaceae & $\begin{array}{c}\text { Cynophalla retusa (Griseb.) } \\
\text { Cornejo \& Iltis }\end{array}$ & - & $\begin{array}{l}\text { G. A. Damasceno- } \\
\text { Junior } 2784\end{array}$ & $\mathrm{COR}$ & FED & 14,19 \\
\hline Caricaceae & Jacaratia corumbensis Kuntze & $\begin{array}{c}\text { Mamãozinho*, } \\
\text { mamãozinho-de-veado (1), } \\
\text { jaracatiá* }\end{array}$ & $\begin{array}{l}\text { G. A. Damasceno- } \\
\text { Junior } 3676\end{array}$ & COR & $\begin{array}{l}\mathrm{CH}, \mathrm{FED} \\
\text { FES }\end{array}$ & $1,2,3$ \\
\hline
\end{tabular}


Quadro 1. Cont.

\begin{tabular}{|c|c|c|c|c|c|c|}
\hline Família & Nome científico & $\begin{array}{l}\text { Nome popular no MS* } \\
\text { "nome indígena"e (fonte) }\end{array}$ & $\begin{array}{l}\text { Nome e Número } \\
\text { do coletor }\end{array}$ & Herbário & Fisionomia & Fontes \\
\hline Caricaceae & $\begin{array}{l}\text { Jacaratia spinosa (Aulbl.) A. } \\
\text { DC }\end{array}$ & $\begin{array}{l}\text { Jaracatiá*, mamãozinho- } \\
\text { do-mato* }\end{array}$ & $\begin{array}{l}\text { G. A. Damasceno- } \\
\text { Junior } 3799\end{array}$ & $\mathrm{COR}$ & $\begin{array}{l}\text { MR, CE, } \\
\text { FED, FES }\end{array}$ & 3,7 \\
\hline Caryocaraceae & Caryocar brasiliense Cambess & Pequi*, piqui* & $\begin{array}{l}\text { I. M. Bortolotto } \\
1209\end{array}$ & COR & $\mathrm{CE}$ & $\begin{array}{l}1,2 \\
3,7\end{array}$ \\
\hline Celastraceae & $\begin{array}{l}\text { Salacia elliptica (Mart. ex } \\
\text { Schult.) G. Don }\end{array}$ & $\begin{array}{l}\text { Siputá*, saputá*, sitobá*, } \\
\text { "mats'î"(Gt, 24) }\end{array}$ & $\begin{array}{l}\text { G. A. Damasceno- } \\
\text { Junior } 357\end{array}$ & $\mathrm{COR}$ & MR, FES & $\begin{array}{c}1,2,5 \\
7,24\end{array}$ \\
\hline Celastraceae & $\begin{array}{c}\text { Peritassa campestris (Cambess.) } \\
\text { A.C. Sm. }\end{array}$ & Bacupari* & A. Pott 14235 & CGMS & $\mathrm{CE}$ & 6 \\
\hline Celastraceae & $\begin{array}{l}\text { Tontelea micrantha (Mart. ex } \\
\text { Schult.) A.C. Sm. }\end{array}$ & $\begin{array}{l}\text { Bacupari-do-cerrado*, } \\
\text { siputá-do-cerrado* }\end{array}$ & A. Pott 10316 & CGMS & $\mathrm{CE}$ & 6 \\
\hline Chrysobalanaceae & $\begin{array}{c}\text { Couepia grandiflora (Mart. \& } \\
\text { Zucc.) Benth. }\end{array}$ & Genciana, suquiana (1) & A. Pott 4376 & CPAP & $\mathrm{CE}$ & 1,2 \\
\hline Chrysobalanaceae & $\begin{array}{l}\text { Couepia uiti (Mart. \& Zucc.) } \\
\text { Benth. ex Hook. f. }\end{array}$ & Fruta-de-pato*, pateiro* & $\begin{array}{l}\text { I. M. Bortolotto } \\
1370\end{array}$ & COR & $\begin{array}{l}\text { MR, CE, } \\
\text { CIP }\end{array}$ & 3,5 \\
\hline Chrysobalanaceae & Parinari obtusifolia Hook. f. & Fruta-de-ema* & A. Pott 14235 & CGMS & $\mathrm{CE}$ & 6 \\
\hline Clusiaceae & $\begin{array}{c}\text { Garcinia gardneriana (Planch. } \\
\text { \& Triana) Zappi }\end{array}$ & $\begin{array}{l}\text { Acupari*, cupari*, } \\
\text { bacupari* }\end{array}$ & $\begin{array}{l}\text { I. M. Bortolotto } \\
1358\end{array}$ & CPAP & MR, FES & $\begin{array}{l}1,2 \\
3,5\end{array}$ \\
\hline Combretaceae & Buchenavia tomentosa Eicher & Tarumarana* & $\begin{array}{l}\text { I. M. Bortolotto } \\
1239\end{array}$ & $\mathrm{COR}$ & $\mathrm{CE}$ & $\begin{array}{l}1,2 \\
3,5\end{array}$ \\
\hline Combretaceae & $\begin{array}{c}\text { Terminalia argentea Mart. et } \\
\text { Zucc. }\end{array}$ & Capitão* & $\begin{array}{l}\text { G. A. Damasceno- } \\
\text { Junior } 1998\end{array}$ & $\mathrm{COR}$ & CE, FES & 1,2 \\
\hline Convolvulaceae & Ipomoea alba $\mathrm{L}$. & - & $\begin{array}{l}\text { G. A. Damasceno- } \\
\text { Junior } 865\end{array}$ & $\mathrm{COR}$ & MR & 1,2 \\
\hline Costaceae & Costus spicatus (Jacq.) Sw. & $\begin{array}{l}\text { Caninha-do-brejo*, cana- } \\
\text { brava* }\end{array}$ & $\begin{array}{l}\text { I. M. Bortolotto } \\
1404\end{array}$ & $\mathrm{COR}$ & CE, FES & 1 \\
\hline Cucurbitaceae & Melancium campestre Naudin & Melancia-do-cerrado & A. Pott 10436 & CGMS & $\mathrm{CE}$ & 6 \\
\hline Dilleniaceae & Curatella americana $\mathrm{L}$. & Lixeira* & $\begin{array}{l}\text { G. A. Damasceno- } \\
\text { Junior } 2985\end{array}$ & COR & $\mathrm{CE}$ & 1,2 \\
\hline Dilleniaceae & $\begin{array}{l}\text { Doliocarpus dentatus (Aubl.) } \\
\text { Standl. }\end{array}$ & Cipó-de-fogo & A. Pott 4316 & CPAP & $\begin{array}{l}\text { MR, CE, } \\
\text { CIP }\end{array}$ & 1,2 \\
\hline Dioscoreaceae & Dioscorea cf. altissima & - & C. Faxina 347 & CGMS & CE, FES & 20 \\
\hline Dioscoreaceae & Dioscorea campestris Griseb. & Cará-do-campo* & $\begin{array}{l}\text { I. M. Bortolotto } \\
1095\end{array}$ & $\mathrm{COR}$ & FED & 0 \\
\hline Dioscoreaceae & Dioscorea corumbensis R. Knuth & - & $\begin{array}{l}\text { G. A. Damasceno- } \\
\text { Junior } 2769\end{array}$ & COR & FED & 0 \\
\hline Dioscoreaceae & Dioscorea dodecaneura Vell. & - & $\begin{array}{l}\text { Hatschbach, G.; } \\
\text { Hatschbach, M. \& } \\
\text { Barbosa, E. } 74703\end{array}$ & INPA & FED & 13,15 \\
\hline Dioscoreaceae & Dioscorea hassleriana Chodat & - & $\begin{array}{l}\text { G. A. Damasceno- } \\
\text { Junior } 2522\end{array}$ & $\mathrm{COR}$ & FED & 15 \\
\hline Dioscoreaceae & Dioscorea ovata Vell. & - & $\begin{array}{l}\text { I. M. Bortolotto } \\
1154\end{array}$ & $\mathrm{COR}$ & FED & 15 \\
\hline Dioscoreaceae & $\begin{array}{l}\text { Dioscorea piperifolia Humb. \& } \\
\text { Bonpl. ex Willd. }\end{array}$ & - & $\begin{array}{l}\text { G. A. Damasceno- } \\
\text { Junior } 2171\end{array}$ & $\mathrm{COR}$ & FED & 15 \\
\hline Dioscoreaceae & Dioscorea trifida L. f. & Japecanga (1) & A. Pott 5335 & CPAP & $\mathrm{CE}$ & 1,2 \\
\hline Ebenaceae & Diospyros hispida A. DC. & $\begin{array}{l}\text { Fruta-de-boi* }{ }^{*} \text {, olho-de } \\
\text { boi (1) }\end{array}$ & A. Pott 4460 & CPAP & $\mathrm{CE}$ & $\begin{array}{l}1,2 \\
5,7\end{array}$ \\
\hline Ebenaceae & Diospyrus obovata Jacq. & Olho-de-boi (1) & A. Pott 2752 & CPAP & $\begin{array}{l}\text { MR, FED, } \\
\text { FES }\end{array}$ & 1,7 \\
\hline Fabaceae & $\begin{array}{c}\text { Arachis appressipila Krapov. \& } \\
\text { W. C. Greg. }\end{array}$ & Amendoim-bravo* & $\begin{array}{l}\text { J.F.M. Valls et al. } \\
\quad 9060\end{array}$ & CEN & CIP & 0 \\
\hline Fabaceae & $\begin{array}{c}\text { Arachis archeri Krapov. \& W.C. } \\
\text { Greg. }\end{array}$ & $\begin{array}{l}\text { Amendoim-do-campo- } \\
\text { limpo* }\end{array}$ & $\begin{array}{l}\text { J.F.M. Valls et al. } \\
7614\end{array}$ & CEN & $\mathrm{CE}$ & 0 \\
\hline Fabaceae & Arachis benthamii Handro & - & $\begin{array}{l}\text { J.F.M. Valls et al. } \\
755\end{array}$ & CEN & CE,MR & 0 \\
\hline
\end{tabular}


Quadro 1. Cont.

\begin{tabular}{|c|c|c|c|c|c|c|}
\hline Família & Nome científico & $\begin{array}{l}\text { Nome popular no MS* } \\
\text { "nome indígena"e (fonte) }\end{array}$ & $\begin{array}{l}\text { Nome e Número } \\
\text { do coletor }\end{array}$ & Herbário & Fisionomia & Fontes \\
\hline Fabaceae & $\begin{array}{c}\text { Arachis brevipetiolata Krapov. } \\
\text { \&W.C.Greg. }\end{array}$ & - & J.F.M. Valls & CEN & $\mathrm{CE}$ & 0 \\
\hline Fabaceae & $\begin{array}{c}\text { Arachis cryptopotamica Krapov. } \\
\text { \& W.C. Greg. }\end{array}$ & - & $\begin{array}{l}\text { A. Krapovickas } \\
\text { \& W.C. Gregory } \\
30023\end{array}$ & CEN & $\mathrm{CE}$ & 0 \\
\hline Fabaceae & Arachis diogoi Hoehne & - & $\begin{array}{l}\text { Damasceno Jr., } \\
\text { G.A. et al. } 2666\end{array}$ & CEN & CIP & 0 \\
\hline Fabaceae & $\begin{array}{c}\text { Arachis douradiana Krapov. \& } \\
\text { W.C. Greg. }\end{array}$ & - & $\begin{array}{l}\text { J.F.M. Valls et al. } \\
\quad 7707\end{array}$ & CEN & $\mathrm{CE}$ & 0 \\
\hline Fabaceae & Arachis glabrata Benth. & $\begin{array}{l}\text { Amendoim-do-campo- } \\
\text { baixo }\end{array}$ & $\begin{array}{l}\text { J.F.M. Valls et al. } \\
\quad 7554\end{array}$ & CEN & CE, MR & 0 \\
\hline Fabaceae & $\begin{array}{c}\text { Arachis gracilis Krapov. \& W.C. } \\
\text { Greg. }\end{array}$ & - & $\begin{array}{l}\text { J.F.M. Valls et al. } \\
\quad 14515\end{array}$ & CEN & CE, MR & 0 \\
\hline Fabaceae & $\begin{array}{c}\text { Arachis guaranitica Chodat \& } \\
\text { Hassl. }\end{array}$ & - & $\begin{array}{l}\text { J.F.M. Valls et al. } \\
\quad 7704\end{array}$ & CEN & $\mathrm{CE}$ & 0 \\
\hline Fabaceae & $\begin{array}{c}\text { Arachis hatschbachii Krapov. \& } \\
\text { W.C. Greg. }\end{array}$ & - & $\begin{array}{l}\text { G. Hatschbach } \\
32105\end{array}$ & MBM & $\mathrm{CE}$ & 0 \\
\hline Fabaceae & $\begin{array}{c}\text { Arachis hermannii Krapov. \& } \\
\text { W.C. Greg. }\end{array}$ & - & $\begin{array}{l}\text { J.F.M. Valls et al. } \\
7555\end{array}$ & CEN & CE, MR & 0 \\
\hline Fabaceae & $\begin{array}{c}\text { Arachis hoehnei Krapov. \& W.C. } \\
\text { Greg. }\end{array}$ & - & $\begin{array}{c}\text { A. } \\
\text { Krapovickas\&W.C } \\
\text { Gregory }\end{array}$ & CEN & $\mathrm{CE}, \mathrm{MR}$ & 0 \\
\hline Fabaceae & Arachis hypogaea $\mathrm{L}$. & Amendoim* & C. S. Bezerra & CGMS & CUL, CE & 25 \\
\hline Fabaceae & $\begin{array}{c}\text { Arachis kretschmeri Krapov. \& } \\
\text { W.C. Greg. }\end{array}$ & - & $\begin{array}{l}\text { J.F.M. Valls et al. } \\
7631\end{array}$ & CEN & CIP, MR & 0 \\
\hline Fabaceae & $\begin{array}{c}\text { Arachis kuhlmannii Krapov. } \\
\text { \&W.C.Greg. }\end{array}$ & - & C. S. Taffarel & CGMS & CE, MR & 0 \\
\hline Fabaceae & $\begin{array}{c}\text { Arachis lignosa (Chodat \& } \\
\text { Hassl.) Krapov. \& W.C. Greg. }\end{array}$ & - & $\begin{array}{l}\text { J.F.M. Valls et al. } \\
\qquad 13570\end{array}$ & CEN & CIP, MR & 0 \\
\hline Fabaceae & $\begin{array}{c}\text { Arachis major Krapov. \&W.C. } \\
\text { Greg. }\end{array}$ & $\begin{array}{l}\text { Amendoim-de- } \\
\text { Aquidauana* }\end{array}$ & $\begin{array}{c}\text { J.F.M. Valls et al. } \\
10407\end{array}$ & CEN & $\mathrm{CE}$ & 0 \\
\hline Fabaceae & Arachis martii Handro & - & $\begin{array}{l}\text { J.F.M. Valls et al. } \\
\quad 15413\end{array}$ & CEN & $\mathrm{CE}$ & 0 \\
\hline Fabaceae & $\begin{array}{c}\text { Arachis microsperma Krapov. et } \\
\text { al. }\end{array}$ & - & $\begin{array}{l}\text { J.F.M. Valls et al. } \\
\quad 768\end{array}$ & CEN & CE & 0 \\
\hline Fabaceae & Arachis nitida Valls et al. & - & $\begin{array}{l}\text { J.F.M. Valls et al. } \\
\qquad 14040\end{array}$ & CEN & $\mathrm{CE}$ & 0 \\
\hline Fabaceae & $\begin{array}{c}\text { Arachis oteroi Krapov. \& W.C. } \\
\text { Greg. }\end{array}$ & - & $\begin{array}{l}\text { J.F.M. Valls et al. } \\
\quad 987\end{array}$ & CEN & $\mathrm{CE}$ & 0 \\
\hline Fabaceae & $\begin{array}{c}\text { Arachis paraguariensis Chodat } \\
\text { \& Hassl. }\end{array}$ & - & $\begin{array}{l}\text { A. Krapovikas } \\
30015\end{array}$ & $\mathrm{RB}$ & $\mathrm{CE}$ & 0 \\
\hline Fabaceae & $\begin{array}{l}\text { Arachis pflugeae C.E. } \\
\text { Simpson et al. }\end{array}$ & - & $\begin{array}{l}\text { J.F.M. Valls et al. } \\
\quad 13589\end{array}$ & CEN & $\mathrm{CI}, \mathrm{CE}$ & 0 \\
\hline Fabaceae & $\begin{array}{l}\text { Arachis pseudovillosa (Chodat \& } \\
\text { Hassl.) Krapov. \& W.C. Greg. }\end{array}$ & - & $\begin{array}{l}\text { J.F.M. Valls et al. } \\
\quad 13593\end{array}$ & CEN & $\mathrm{CE}$ & 0 \\
\hline Fabaceae & Arachis repens Handro & - & $\begin{array}{l}\text { Hatschbach G. et } \\
\quad \text { al. } 59057\end{array}$ & MBM & $\mathrm{CE}$ & 0 \\
\hline Fabaceae & $\begin{array}{c}\text { Arachis stenophylla Krapov. \& } \\
\text { W.C. Greg. }\end{array}$ & - & $\begin{array}{l}\text { J.F.M. Valls et al. } \\
\qquad 14026\end{array}$ & CEN & $\mathrm{CE}$ & 0 \\
\hline Fabaceae & Arachis tuberosa Bong. ex Benth. & - & $\begin{array}{l}\text { J.F.M. Valls et al. } \\
\qquad 14632\end{array}$ & CEN & $\mathrm{CE}$ & 0 \\
\hline Fabaceae & $\begin{array}{c}\text { Arachis valida Krapov. \& } \\
\text { W.C.Greg. }\end{array}$ & - & $\begin{array}{l}\text { J.F.M. Valls et al. } \\
\quad 9153\end{array}$ & CEN & CIP & 0 \\
\hline Fabaceae & $\begin{array}{c}\text { Arachis vallsii Krapov. \& } \\
\text { W.C.Greg. }\end{array}$ & - & $\begin{array}{l}\text { J.F.M. Valls et al. } \\
\quad 7635\end{array}$ & CEN & CIP & 0 \\
\hline Fabaceae & $\begin{array}{c}\text { Canavalia } \\
\text { mattogrossensis (Barb. Rodr.) } \\
\text { Malme }\end{array}$ & $\begin{array}{l}\text { Feijão-bravo (1), feijão-do- } \\
\text { mato (1), faveirinho ( } 1\end{array}$ & A. Pott 4433 & CPAP & MR & 1 \\
\hline
\end{tabular}


Quadro 1. Cont.

\begin{tabular}{|c|c|c|c|c|c|c|}
\hline Família & Nome científico & $\begin{array}{l}\text { Nome popular no MS* } \\
\text { “nome indígena"e (fonte) }\end{array}$ & $\begin{array}{l}\text { Nome e Número } \\
\text { do coletor }\end{array}$ & Herbário & Fisionomia & Fontes \\
\hline Fabaceae & Cassia grandis L. f. & Canafístula* & I. M. Bortolotto 922 & COR & MR, FES & 5 \\
\hline Fabaceae & Copaifera coriacea Mart. & Guranazinho (1) & A. Pott 3237 & CPAP & $\mathrm{CE}$ & 1,2 \\
\hline Fabaceae & Dipteryx alata Vogel & $\begin{array}{l}\text { Cumbaru*, baru*, } \\
\text { castanha-do- cerrado* }\end{array}$ & $\begin{array}{l}\text { G. A. Damasceno- } \\
\text { Junior } 3809\end{array}$ & COR & $\mathrm{CE}$ & $1,2,7$ \\
\hline Fabaceae & Discolobium pulchellum Benth. & Cortiça (2) & V. J. Pott 1037 & CPAP & CIP & 2,4 \\
\hline Fabaceae & Geoffroea spinosa Jacq. & Mani (2) & $\begin{array}{l}\text { M.V. Martins } 215 \\
\text { CGMS }\end{array}$ & CGMS & $\mathrm{CH}$ & 2,5 \\
\hline Fabaceae & Hymenaea courbaril L. & Jatobá-mirim* & $\begin{array}{l}\text { G. A. Damasceno- } \\
\text { Junior } 439\end{array}$ & COR & $\mathrm{MR}, \mathrm{CE}$ & $1,2,3$ \\
\hline Fabaceae & Hymenaea martiana Hayne & Jatobá*; jatobá-mirim* & S.R. Zacharias 338 & CGMS & $\begin{array}{l}\text { MR, CE, } \\
\text { FES }\end{array}$ & 5 \\
\hline Fabaceae & $\begin{array}{c}\text { Hymenaea stigonocarpa Mart. } \\
\text { ex Hayne }\end{array}$ & $\begin{array}{l}\text { Jatobá*, jatobero (1), } \\
\text { jatobá-do-cerrado (1) }\end{array}$ & $\begin{array}{l}\text { G. A. Damasceno- } \\
\text { Junior } 4572\end{array}$ & COR & $\mathrm{CE}$ & $1,2,3$ \\
\hline Fabaceae & Inga laurina (Sw.) Willd. & - & $\begin{array}{l}\text { I. M. Bortolotto } \\
1600\end{array}$ & COR & $\mathrm{CE}$ & 5 \\
\hline Fabaceae & $\begin{array}{l}\text { Inga oerstediana Benth. ex } \\
\text { Seem. }\end{array}$ & - & $\begin{array}{l}\text { G.M. Silva; A.L. } \\
\text { B. Sartori } 15158\end{array}$ & CGMS & MR & 15 \\
\hline Fabaceae & Inga marginata Willd. & - & A. Pott 12405 & CGMS & $\mathrm{MR}, \mathrm{CE}$ & 6 \\
\hline Fabaceae & Inga semialata (Vell.) Mart. & Ingá-feijão & $\begin{array}{l}\text { G. A. Damasceno- } \\
\text { Junior } 4489\end{array}$ & COR & $\mathrm{MR}, \mathrm{CE}$ & 0 \\
\hline Fabaceae & Inga striata Benth. & - & J.P. Ramos 10 & CGMS & & 15 \\
\hline Fabaceae & Inga vera Willd. & Ingá* & $\begin{array}{l}\text { G. A. Damasceno- } \\
\text { Junior } 1439\end{array}$ & CPAP & MR & $\begin{array}{l}1,2,3 \\
5,7\end{array}$ \\
\hline Fabaceae & Phaseolus lunatus L. & Feijãozinho*, feijão-fava* & A. Pott 3068 & CGMS & $\mathrm{CH}$ & 6 \\
\hline Fabaceae & Prosopis ruscifolia Griseb. & Algarobo* & $\begin{array}{l}\text { G. A. Damasceno- } \\
\text { Junior } 5142\end{array}$ & COR & $\mathrm{MR}, \mathrm{CH}$ & 1,2 \\
\hline Fabaceae & $\begin{array}{l}\text { Samanea tubulosa (Benth.) } \\
\text { Barneby \& J.W. Grimes }\end{array}$ & Farinha-seca* & $\begin{array}{l}\text { G. A. Damasceno- } \\
\text { Junior } 3725\end{array}$ & COR & FED, FES & 1 \\
\hline Fabaceae & Senna occidentalis (L.) Link & Fedegoso* & $\begin{array}{l}\text { G. A. Damasceno- } \\
\text { Junior } 1091\end{array}$ & COR & CIP, RU & $1,2,3,5$ \\
\hline Icacinaceae & Emmotum nitens (Benth.) Miers & Sobre* & A. Pott 13162 & CGMS & $\mathrm{CE}, \mathrm{MR}$ & 6 \\
\hline Lamiaceae & Vitex cymosa Bertero ex Spreng. & Tarumã*, "madô"” (Gt, 24) & $\begin{array}{l}\text { G. A. Damasceno- } \\
\text { Junior } 373\end{array}$ & COR & MR & $\begin{array}{l}1,2,3 \\
5,7,24\end{array}$ \\
\hline Lecythidaceae & Eschweilera nana (O. Berg) & Ovo-frito* & A. Pott 12814 & CGMS & $\mathrm{CE}$ & 6 \\
\hline Loganiaceae & $\begin{array}{c}\text { Strychnos pseudoquina A. } \\
\text { St.-Hil. }\end{array}$ & Limãozinho* & A. Pott 11475 & CGMS & $\mathrm{CE}$ & 6 \\
\hline Malpighiaceae & $\begin{array}{c}\text { Byrsonima aff. arthropoda A. } \\
\text { Juss. }\end{array}$ & Canjicão* & $\begin{array}{l}\text { Tozzi, A. M. G. } \\
\text { A. } 143\end{array}$ & COR & MR & 3 \\
\hline Malpighiaceae & Byrsonima coccolobifolia Kunth & Canjiqueira* & $\begin{array}{l}\text { I. M. Bortolotto } \\
1204\end{array}$ & COR & $\mathrm{CE}$ & 5 \\
\hline Malpighiaceae & Byrsonima crassifolia (L.) Kunth & Canjicão* & A. Pott 3809 & CPAP & $\mathrm{CE}, \mathrm{FES}$ & $1,2,7$ \\
\hline Malpighiaceae & Byrsonima intermedia A. Juss. & Murici-do-campo* & A. Pott 11512 & CGMS & $\mathrm{CE}$ & 6 \\
\hline Malpighiaceae & $\begin{array}{c}\text { Byrsonima verbascifolia (L.) } \\
\text { DC. }\end{array}$ & Murici* & A. Pott 2391 & CPAP & $\mathrm{CE}$ & $1,2,7$ \\
\hline Malpighiaceae & Byrsonima cydoniifolia A. Juss. & $\begin{array}{l}\text { Canjiqueira*, canjiquinha*, } \\
\text { canjica (1), murici (1) }\end{array}$ & A. Pott 5049 & CPAP & MR, CIP & $1,2,3,5$ \\
\hline Malvaceae & $\begin{array}{c}\text { Eriotheca roseorum (Cuatrec.) } \\
\text { A. Robyns }\end{array}$ & Imbirussu* & $\begin{array}{l}\text { G. A. Damasceno- } \\
\text { Junior } 1905\end{array}$ & COR & FED, FES & 6 \\
\hline Malvaceae & Guazuma ulmifolia Lam. & $\begin{array}{c}\text { Chico-magro } * \text {, mutambo } \\
\text { (1) }\end{array}$ & $\begin{array}{l}\text { G. A. Damasceno- } \\
\text { Junior } 3681\end{array}$ & COR & $\begin{array}{l}\text { MR, CE, } \\
\text { FED, FES }\end{array}$ & 1 \\
\hline Malvaceae & $\begin{array}{c}\text { Sterculia apetala (Jacq.) H. } \\
\text { Karst }\end{array}$ & $\begin{array}{l}\text { Mandovi*, manduvi*, } \\
\text { manduvizeiro (1), } \\
\text { amendoim-de-bugre (1) }\end{array}$ & $\begin{array}{l}\text { G. A. Damasceno- } \\
\text { Junior } 563\end{array}$ & COR & FED, FES & $\begin{array}{l}1,2 \\
3,7\end{array}$ \\
\hline Malvaceae & Sterculia striata St. Hil. Et Naud. & Manduvi* & $\begin{array}{l}\text { I. M. Bortolotto } \\
1288\end{array}$ & COR & FED & 3,7 \\
\hline Marantaceae & Thalia geniculata L. & Caeté* & V. j. Pott 2289 & CPAP & CIP & 4 \\
\hline
\end{tabular}


Quadro 1. Cont.

\begin{tabular}{|c|c|c|c|c|c|c|}
\hline Família & Nome científico & $\begin{array}{l}\text { Nome popular no MS* } \\
\text { “nome indígena"e (fonte) }\end{array}$ & $\begin{array}{l}\text { Nome e Número } \\
\text { do coletor }\end{array}$ & Herbário & Fisionomia & Fontes \\
\hline Melastomataceae & Clidemia cf. biserrata DC. & Cambucá-do-firme* & $\begin{array}{l}\text { G. A. Damasceno- } \\
\text { Junior } 2926\end{array}$ & COR & $\mathrm{MR}, \mathrm{CE}$ & 3 \\
\hline Melastomataceae & Miconia chamissois Naudin & Sabiazeira & A. Pott 4828 & CGMS & $\mathrm{CI}$ & 6 \\
\hline Melastomataceae & Mouriri elliptica Mart. & Coroa-de-frade, coroa (1) & $\begin{array}{l}\text { G. A. Damasceno- } \\
\text { Junior } 1933\end{array}$ & COR & $\mathrm{CE}$ & $1,2,3$ \\
\hline Melastomataceae & Mouriri guianensis Aubl. & Roncador* & $\begin{array}{l}\text { I. M. Bortolotto } \\
912\end{array}$ & COR & MR & $1,2,5$ \\
\hline Menispermaceae & $\begin{array}{l}\text { Abuta grandifolia ( Mart.) } \\
\text { Sandwith }\end{array}$ & $\begin{array}{l}\text { Grão-de-galo*, } \\
\text { manguinha* }\end{array}$ & $\begin{array}{l}\text { I. M. Bortolotto } \\
1173\end{array}$ & COR & MR, FES & 3,5 \\
\hline Menispermaceae & Disciphania ernstii Eichler & Uva-do-mato* & $\begin{array}{l}\text { G. A. Damasceno- } \\
\text { Junior } 2866\end{array}$ & COR & FED, FES & 3 \\
\hline Menyanthaceae & Nymphoides indica (L.) Kuntze & Lagartixa & A. Pott 6868 & CPAP & CIP & 4 \\
\hline Moraceae & Brosimum gaudichaudii Trécul & $\begin{array}{l}\text { Mama-cadela, } \\
\text { algodãozinho (1) }\end{array}$ & A. Pott 4965 & CPAP & $\begin{array}{l}\mathrm{MR}, \mathrm{CH} \\
\mathrm{CE}, \mathrm{FES}\end{array}$ & $1,2,3$ \\
\hline Moraceae & Ficus pertusa L.f. & $\begin{array}{l}\text { Fiueirinha* ou figueira- } \\
\text { de-folha miúda (2) }\end{array}$ & $\begin{array}{l}\text { G. A. Damasceno- } \\
\text { Junior } 530\end{array}$ & COR & MR, FES & 2 \\
\hline Moraceae & $\begin{array}{c}\text { Maclura tinctoria (L.) D. Don } \\
\text { ex Steud }\end{array}$ & $\begin{array}{c}\text { Taiúva*, mora, amora- } \\
\text { brava, moreira, amora-do- } \\
\text { mato }(1)\end{array}$ & $\begin{array}{l}\text { I. M. Bortolotto } \\
1168\end{array}$ & COR & FED, FES & $1,2,5$ \\
\hline Moraceae & $\begin{array}{c}\text { Sorocea sprucei (Baill.) J.F. } \\
\text { Macbr. }\end{array}$ & $\begin{array}{l}\text { Figueirinha, figueirinha- } \\
\text { do-Pantanal, leiteiro- } \\
\text { branco (1) }\end{array}$ & A. Pott 3609 & CPAP & MR, FES & 1 \\
\hline Myrtaceae & $\begin{array}{l}\text { Blepharocalyx salicifolius } \\
\text { (Kunth) O. Berg }\end{array}$ & - & $\begin{array}{l}\text { I. M. Bortolotto } \\
216\end{array}$ & COR & CE, FES & 13,18 \\
\hline Myrtaceae & $\begin{array}{c}\text { Calyptranthes lucida Mart. ex } \\
\text { DC. }\end{array}$ & - & $\begin{array}{l}\text { G. A. Damasceno- } \\
\text { Junior } 2616\end{array}$ & COR & $\mathrm{MR}$ & 13 \\
\hline Myrtaceae & $\begin{array}{l}\text { Campomanesia adamantium } \\
\text { (Cambess.) O. Berg }\end{array}$ & Guavira* & $\begin{array}{l}\text { G. A. Damasceno- } \\
\text { Junior } 4013\end{array}$ & COR & $\mathrm{CE}$ & 3 \\
\hline Myrtaceae & $\begin{array}{c}\text { Campomanesia eugenioides } \\
\text { (Cambess.) D. Legrand ex } \\
\text { Landrum }\end{array}$ & Guavira* & A. Pott 5248 & CPAP & MR & 3 \\
\hline Myrtaceae & $\begin{array}{l}\text { Campomanesia pubescens } \\
\text { (Mart. ex DC.) O. Berg }\end{array}$ & Guavira* & $\begin{array}{l}\text { G. A. Damasceno- } \\
\text { Junior } 920\end{array}$ & COR & $\mathrm{CE}$ & 3 \\
\hline Myrtaceae & $\begin{array}{c}\text { Campomanesia sessiliflora (O. } \\
\text { Berg) Mattos }\end{array}$ & Guavira* & $\begin{array}{l}\text { G. A. Damasceno- } \\
\text { Junior } 4470\end{array}$ & COR & $\mathrm{CE}$ & 3 \\
\hline Myrtaceae & $\begin{array}{c}\text { Campomanesia xanthocarpa } \\
\text { (Mart.) O. Berg }\end{array}$ & Guavira* & $\begin{array}{l}\text { G. A. Damasceno- } \\
\text { Junior } 1634\end{array}$ & COR & MR & 3 \\
\hline Myrtaceae & Eugenia aurata O. Berg & - & $\begin{array}{l}\text { G. A. Damasceno- } \\
\text { Junior } 3321\end{array}$ & CPAP & CE, FED & 1 \\
\hline Myrtaceae & Eugenia bimarginata DC. & - & A. Pott 11341 & CPAP & $\mathrm{CE}$ & 0 \\
\hline Myrtaceae & Eugenia calycina Cambess. & Cereja-do-cerrado* & $\begin{array}{c}\text { Caxambu, M. G. } \\
2297\end{array}$ & MBM & $\mathrm{CE}$ & 3 \\
\hline Myrtaceae & Eugenia dysenterica (Mart.) DC. & Cagaita* & $\begin{array}{l}\text { G. A. Damasceno- } \\
\text { Junior } 1066\end{array}$ & COR & $\mathrm{CE}$ & 3 \\
\hline Myrtaceae & Eugenia egensis DC. & - & $\begin{array}{l}\text { G. A. Damasceno- } \\
\text { Junior } 1066\end{array}$ & COR & MR & 1 \\
\hline Myrtaceae & Eugenia florida DC. & Jamelão-do-campo (2) & $\begin{array}{l}\text { G. A. Damasceno- } \\
\text { Junior } 1622\end{array}$ & COR & MR, FES & 1,2 \\
\hline Myrtaceae & Eugenia inundata DC. & - & A. Pott 5311 & CPAP & MR & 1 \\
\hline Myrtaceae & Eugenia moraviana O. Berg & - & $\begin{array}{l}\text { G. A. Damasceno- } \\
\text { Junior } 2003\end{array}$ & COR & MR, FES & 0 \\
\hline Myrtaceae & $\begin{array}{c}\text { Eugenia pseudoverticillata } \mathrm{S} . \\
\text { Moore }\end{array}$ & - & $\begin{array}{l}\text { G. A. Damasceno- } \\
\text { Junior } 478\end{array}$ & COR & MR & 0 \\
\hline Myrtaceae & $\begin{array}{c}\text { Eugenia punicifolia (Kunth.) } \\
\text { DC. }\end{array}$ & - & $\begin{array}{l}\text { G. A. Damasceno- } \\
\text { Junior } 3437\end{array}$ & COR & $\mathrm{CE}$ & 21 \\
\hline
\end{tabular}


Quadro 1. Cont.

\begin{tabular}{|c|c|c|c|c|c|c|}
\hline Família & Nome científico & $\begin{array}{l}\text { Nome popular no MS* } \\
\text { "nome indígena"e (fonte) }\end{array}$ & $\begin{array}{l}\text { Nome e Número } \\
\text { do coletor }\end{array}$ & Herbário & Fisionomia & Fontes \\
\hline Myrtaceae & Eugenia pyriformis Cambess. & - & $\begin{array}{l}\text { G. A. Damasceno- } \\
\text { Junior } 1974\end{array}$ & $\mathrm{COR}$ & MR, FES & $1,2,3$ \\
\hline Myrtaceae & Eugenia racemulosa $\mathrm{O}$. Berg & Cambucá* & C.A.Conceição & COR & MR & 25 \\
\hline Myrtaceae & Eugenia repanda $\mathrm{O}$. Berg & - & $\begin{array}{l}\text { G. A. Damasceno- } \\
\text { Junior } 4077\end{array}$ & $\mathrm{COR}$ & FED & 0 \\
\hline Myrtaceae & Eugenia subterminalis DC. & - & $\begin{array}{l}\text { G. A. Damasceno- } \\
\text { Junior } 3608\end{array}$ & $\mathrm{COR}$ & MR & 0 \\
\hline Myrtaceae & Eugenia tapacumensis O. Berg & $\begin{array}{l}\text { Cambucá, língua-de- } \\
\text { cachorro (1) }\end{array}$ & A. Pott 5634 & CPAP & CE, FES & 1,2 \\
\hline Myrtaceae & Eugenia uniflora L. & Pitanga* & P.V. Gil 1 (CGMS) & CGMS & $\mathrm{CE}$ & 21 \\
\hline Myrtaceae & Eugenia matogrossensis Sobral & Guabiroba* & $\begin{array}{l}\text { G. A. Damasceno- } \\
\text { Junior } 411\end{array}$ & $\mathrm{COR}$ & $\mathrm{CE}$ & 0 \\
\hline Myrtaceae & $\begin{array}{l}\text { Gomidesia palustris (DC.) } \\
\text { Kaussel }\end{array}$ & Balsemim (1) & A. Pott 5476 & CPAP & $\mathrm{CE}$ & 1,2 \\
\hline Myrtaceae & $\begin{array}{l}\text { Hexachlamys edulis }(\mathrm{O} . \text { Berg}) \\
\text { Kausel \& D. Legrand }\end{array}$ & - & $\begin{array}{l}\text { G. A. Damasceno- } \\
\text { Junior } 3773\end{array}$ & $\mathrm{COR}$ & & 3 \\
\hline Myrtaceae & Myrcia fallax (Rich.) DC. & "Miguá" (Gt, 24) & A. Pott 4925 & CPAP & MR & 1,24 \\
\hline Myrtaceae & $\begin{array}{l}\text { Myrcianthes pungens (O. Berg) } \\
\text { D. Legrand }\end{array}$ & Guabijú* & $\begin{array}{l}\text { G. A. Damasceno- } \\
\text { Junior } 1655\end{array}$ & COR & MR & 13 \\
\hline Myrtaceae & Plinia cauliflora (Mart.) Kausel & Jabuticaba* & $\begin{array}{l}\text { I. M. Bortolotto } \\
1654\end{array}$ & $\mathrm{COR}$ & FED & 3,5 \\
\hline Myrtaceae & Psidium acutangulum DC. & Araçá* & $\begin{array}{l}\text { G. A. Damasceno- } \\
\text { Junior } 2357\end{array}$ & $\mathrm{COR}$ & MR & 3 \\
\hline Myrtaceae & Psidium guineense $\mathrm{Sw}$. & Araçá, goiaba (1) & $\begin{array}{l}\text { G. A. Damasceno- } \\
\text { Junior } 2917\end{array}$ & CPAP & MR, FES & 1,2 \\
\hline Myrtaceae & Psidium kennedyanum Morong & Araçazinho (1) & A. Pott 3177 & CPAP & MR & 1 \\
\hline Myrtaceae & Psidium nutans O. Berg. & - & $\begin{array}{l}\text { G. A. Damasceno- } \\
\text { Junior } 4278\end{array}$ & COR & FES & 0 \\
\hline Myrtaceae & Psidium persicifolium $\mathrm{O}$. Berg & Goiabinha & $\begin{array}{l}\text { G. A. Damasceno- } \\
\text { Junior } 2658 \text { A }\end{array}$ & $\mathrm{COR}$ & MR & 22 \\
\hline Myrtaceae & $\begin{array}{l}\text { Psidium sartorianum (O. Berg) } \\
\text { Nied. }\end{array}$ & Araçá* & $\begin{array}{l}\text { G. A. Damasceno- } \\
\text { Junior } 3600\end{array}$ & $\mathrm{COR}$ & MR, FED & 22 \\
\hline Myrtaceae & Psidium kennedyanum Morong & $\begin{array}{c}\text { Araçá-bravo, araçazinho } \\
\text { (1) }\end{array}$ & I. M. Bortolotto 95 & $\mathrm{COR}$ & $\begin{array}{l}\mathrm{MR}, \mathrm{CH} \\
\mathrm{CE}, \mathrm{CIP}\end{array}$ & 1,22 \\
\hline Myrtaceae & Psidium laruotteanum Cambess. & Araçá* & $\begin{array}{l}\text { G. A. Damasceno- } \\
\text { Junior } 1836\end{array}$ & $\mathrm{COR}$ & $\mathrm{CE}$ & 6 \\
\hline Myrtaceae & Stenocalyx pitanga $\mathrm{O}$. Berg & $\begin{array}{l}\text { Pitanga*, pitanguinha, } \\
\text { pitangueira (1) }\end{array}$ & $\begin{array}{l}\text { G. A. Damasceno- } \\
\text { Junior } 478\end{array}$ & CPAP & FES & $\begin{array}{c}1,2,3, \\
15\end{array}$ \\
\hline Nyctaginaceae & Neea hermaphrodita S. Moore & Pau-de-sal (1) & $\begin{array}{l}\text { G. A. Damasceno- } \\
\text { Junior } 309\end{array}$ & COR & MR & 1,2 \\
\hline Nymphaeaceae & $\begin{array}{c}\text { Nymphaea amazonum Mart. \& } \\
\text { Zucc. }\end{array}$ & - & V. J. Pott 2957 & CPAP & CIP & 4 \\
\hline Nymphaeaceae & $\begin{array}{c}\text { Victoria amazonica (Poepp.) J.C. } \\
\text { Sowerby }\end{array}$ & $\begin{array}{l}\text { Vitória-régia *, forno- } \\
\text { d'água* }\end{array}$ & V. J. Pott 1999 & CPAP & CIP & 4,5 \\
\hline Opiliaceae & $\begin{array}{c}\text { Agonandra brasiliensis Miers ex } \\
\text { Benth. \& Hook. f. }\end{array}$ & Tinge-cuia (1) & A. Pott 4476 & CPAP & CE, FES & $1,2,3$ \\
\hline Orchidaceae & $\begin{array}{l}\text { Vanilla palmarum (Salzm. ex } \\
\text { Lindl.) Lindl. }\end{array}$ & Baunilha-de-acuri (1) & A. Pott 5045 & CPAP & MR & 1 \\
\hline Pteridaceae & $\begin{array}{c}\text { Ceratopteris pteridoides (Hook.) } \\
\text { Hieron. }\end{array}$ & - & V. J. Pott 369 & CPAP & CIP & 1,2 \\
\hline Passifloraceae & Passiflora alata Curtis & - & A. Pott 11864 & UPCB & $\mathrm{CE}$ & 23 \\
\hline Passifloraceae & $\begin{array}{c}\text { Passiflora amethystina } \mathrm{J} \\
\text {.C.Mikan }\end{array}$ & Maracujá* & $\begin{array}{l}\text { A.C. Conceição } \\
2736\end{array}$ & $\mathrm{COR}$ & CE, CIP & 18 \\
\hline Passifloraceae & Passiflora cincinnata Mast. & Maracujá-do-mato* & $\begin{array}{l}\text { I. M. Bortolotto } \\
784\end{array}$ & COR & $\mathrm{CE}$ & $3,5,7$ \\
\hline Passifloraceae & Passiflora edulis Sims & Maracujá* & $\begin{array}{l}\text { Hatschbach, G. } \\
\quad 58875\end{array}$ & MBM & CUL, CE & 6 \\
\hline
\end{tabular}


Quadro 1. Cont.

\begin{tabular}{|c|c|c|c|c|c|c|}
\hline Família & Nome científico & $\begin{array}{l}\text { Nome popular no MS* } \\
\text { "nome indígena"e (fonte) }\end{array}$ & $\begin{array}{l}\text { Nome e Número } \\
\text { do coletor }\end{array}$ & Herbário & Fisionomia & Fontes \\
\hline Passifloraceae & $\begin{array}{l}\text { Passiflora foetida var. hispida } \\
\text { (DC.) Killip ex Gleason }\end{array}$ & Maracujá-do-mato* & $\begin{array}{l}\text { G. A. Damasceno- } \\
\text { Junior } 1016\end{array}$ & COR & MR & 11 \\
\hline Passifloraceae & Passiflora gibertii N.E. Br. & $\begin{array}{c}\text { Maracujazinho*, maracujá } \\
\text { bravo*, maracujá-do- } \\
\text { mato* }\end{array}$ & $\begin{array}{l}\text { I. M. Bortolotto } \\
594\end{array}$ & $\mathrm{COR}$ & MR, FED & $3,5,7$ \\
\hline Passifloraceae & Passiflora mansoi (Mart.) Mast. & - & V.J. Pott 4688 & UPCB & $\mathrm{CE}$ & 0 \\
\hline Passifloraceae & Passiflora speciosa Gardner & - & A. Pott 8600 & UPCB & FED & 0 \\
\hline Passifloraceae & Passiflora tricuspis Mast. & - & $\begin{array}{l}\text { G. A. Damasceno- } \\
\text { Junior } 3138\end{array}$ & COR & $\mathrm{CE}$ & 0 \\
\hline Passifloraceae & Passiflora capsularis L. & - & A. Pott 8515 & $\begin{array}{r}\text { UPCI } \\
53654\end{array}$ & FED & 0 \\
\hline Passifloraceae & Passiflora chrysophylla Chodat & - & A. POTT 1723 & CPAP & FES & 0 \\
\hline Passifloraceae & Passiflora suberosa $\mathrm{L}$. & - & A. Pott 11712 & UPCB & FED, CE & 0 \\
\hline Piperaceae & Piper aduncum L.var. aduncum & Pimenta-do-mato* & $\begin{array}{l}\text { G. A. Damasceno- } \\
\text { Junior } 3789\end{array}$ & $\mathrm{COR}$ & MR, FES & 1,2 \\
\hline Piperaceae & $\begin{array}{l}\text { Piper arboreum subsp. } \\
\text { tuberculatum (Jacq.) Tebbs }\end{array}$ & $\begin{array}{c}\text { Pimenta-do-mato, } \\
\text { pimenta-de-macaco, dedo- } \\
\text { de-urubu (1) }\end{array}$ & A. Pott 2711 & CPAP & FES & 1,2 \\
\hline Piperaceae & Piper fuligineum Kunth & - & V.J. Pott 6442 & CGMS & $\mathrm{CI}$ & 6 \\
\hline Poaceae & $\begin{array}{l}\text { Guadua chacoensis (Rojas) } \\
\text { Londoño \& P.M. Peterson }\end{array}$ & Taquaruçu*, taquara* & S.R. Zacharias 408 & CGMS & MR & 0 \\
\hline Poaceae & $\begin{array}{l}\text { Homalocenchrus hexandrus } \\
\text { (Sw.) Kuntze }\end{array}$ & - & V.J. Pott 3137 & CPAP & CIP & 4 \\
\hline Poaceae & Oryza latifolia Desv. & $\begin{array}{l}\text { Arroz-do-campo*, capim- } \\
\text { arroz", "matchamo" (Gt, 24) }\end{array}$ & V.J. Pott 1763 & CPAP & CIP, & $\begin{array}{c}3,4,5 \\
6,24\end{array}$ \\
\hline Poaceae & Oryza glumaepatula Steud. & $\begin{array}{l}\text { Arroz-do-brejo*, capim- } \\
\text { arroz*, arroz-bravo*, } \\
\text { "matchamo"(Gt, 24) }\end{array}$ & $\begin{array}{l}\text { G. A. Damasceno- } \\
\text { Junior } 5139\end{array}$ & $\mathrm{COR}$ & CIP, & $\begin{array}{c}2,3,4 \\
524\end{array}$ \\
\hline Polygonaceae & Coccoloba parimensis Benth. & $\begin{array}{c}\text { Canjiquinha* } \text {, uveira-do- } \\
\text { mato, uvinha, rosarinho, } \\
\text { uvinha (1) }\end{array}$ & $\begin{array}{l}\text { I. M. Bortolotto } \\
1077\end{array}$ & $\mathrm{COR}$ & MR & $1,2,5$ \\
\hline Polygonaceae & $\begin{array}{l}\text { Salta triflora(Griseb.) Adr. } \\
\text { Sanchez }\end{array}$ & - & $\begin{array}{l}\text { G. A. Damasceno- } \\
\text { Junior } 2813\end{array}$ & $\mathrm{COR}$ & MR & 11 \\
\hline Pontederiaceae & $\begin{array}{c}\text { Eichhornia crassipes (Mart.) } \\
\text { Solms. }\end{array}$ & Camalote*, Aguapé* & V.J. Pott 3133 & CPAP & CIP, CI & 2,4 \\
\hline Pontederiaceae & $\begin{array}{c}\text { Hydrocharis dubia (Blume) } \\
\text { Backer }\end{array}$ & $\begin{array}{l}\text { Guapé, aguapé, camalote, } \\
\text { lanceiro, espigácea (4) }\end{array}$ & N. C. Bueno 289 & CPAP & CIP & 4 \\
\hline Portulacaceae & Portulaca fluvialis D. Legrand & $\begin{array}{c}\text { Nove-horas*, nove-hora*, } \\
\text { onze-horas (1) }\end{array}$ & A. Pott 3961 & CPAP & CIP & 1,2 \\
\hline Rhamnaceae & $\begin{array}{c}\text { Rhamnidium elaeocarpum } \\
\text { Reissek }\end{array}$ & Cabriteira* & $\begin{array}{l}\text { G. A. Damasceno- } \\
\text { Junior } 265\end{array}$ & $\mathrm{COR}$ & $\mathrm{MR}, \mathrm{CE}$ & $1,2,5$ \\
\hline Rhamnaceae & Zizyphus oblongifolius S. Moore & $\begin{array}{l}\text { Veludinho*, olho-de-boi*, } \\
\text { "macariguá " }(\mathrm{Gt}, 24)\end{array}$ & $\begin{array}{l}\text { I. M. Bortolotto } \\
737\end{array}$ & $\mathrm{COR}$ & $\begin{array}{l}\text { MR, CH, } \\
\text { FED }\end{array}$ & $1,5,24$ \\
\hline Rubiaceae & Alibertia edulis (Rich.) A. Rich. & Marmelo* & $\begin{array}{l}\text { I. M. Bortolotto } \\
1031\end{array}$ & $\mathrm{COR}$ & $\begin{array}{l}\text { MR, CE } \\
\text { FES }\end{array}$ & $\begin{array}{l}1,2,3 \\
5,7\end{array}$ \\
\hline Rubiaceae & Cordiera sessilis (Vell.) Kuntze & $\begin{array}{l}\text { Marmelada, marmelada- } \\
\text { preta, marmelada-de- } \\
\text { cachorro (1) }\end{array}$ & $\begin{array}{l}\text { I. M. Bortolotto } \\
1530\end{array}$ & $\mathrm{COR}$ & $\begin{array}{l}\text { MR, CE, } \\
\text { FES }\end{array}$ & $1,2,3$ \\
\hline Rubiaceae & $\begin{array}{c}\text { Coussarea } \\
\text { hydrangeifolia (Benth.) Müll. } \\
\text { Arg. }\end{array}$ & Fruta-de-anta & A. Pott 12545 & CGMS & FES & 6 \\
\hline Rubiaceae & Genipa americana $\mathrm{L}$. & Jenipapo,* “mató" (Gt, 24) & $\begin{array}{l}\text { G. A. Damasceno- } \\
\text { Junior } 334\end{array}$ & $\mathrm{COR}$ & MR, FES & $\begin{array}{l}1,2,3 \\
5,7,24\end{array}$ \\
\hline Rubiaceae & $\begin{array}{c}\text { Pentodon pentandrus } \\
\text { (Schumach. \& Thonn.) Vatke }\end{array}$ & - & V.J. Pott 749 & CPAP & CIP & 4 \\
\hline Rubiaceae & $\begin{array}{l}\text { Randia ferox (Cham. \& Schltdl.) } \\
\text { DC. }\end{array}$ & $\begin{array}{l}\text { Veludo-de-espinho, } \\
\text { espinheiro, unha-de-gato } \\
\text { (1) }\end{array}$ & $\begin{array}{l}\text { G. A. Damasceno- } \\
\text { Junior } 599\end{array}$ & COR & MR, FES & 1,2 \\
\hline Rubiaceae & $\begin{array}{c}\text { Rudgea viburnoides (Cham.) } \\
\text { Benth. }\end{array}$ & Veludo & A. Pott 9580 & CGMS & MR, FES & 6 \\
\hline
\end{tabular}


Quadro 1. Cont.

\begin{tabular}{|c|c|c|c|c|c|c|}
\hline Família & Nome científico & $\begin{array}{l}\text { Nome popular no MS* } \\
\text { "nome indígena"e (fonte) }\end{array}$ & $\begin{array}{c}\text { Nome e Número } \\
\text { do coletor }\end{array}$ & Herbário & Fisionomia & Fontes \\
\hline Rutaceae & Esenbeckia almawillia Kaastra & Côca* & $\begin{array}{l}\text { I. M. Bortolotto } \\
1237\end{array}$ & COR & FED & 5 \\
\hline Salicaceae & Casearia rupestris Eichler & Pururuca & $\begin{array}{l}\text { G. A. Damasceno- } \\
\text { Junior } 2497\end{array}$ & $\mathrm{COR}$ & CE, FES & 7 \\
\hline Salicaceae & Casearia sylvestris $\mathrm{Sw}$. & Chá-de-frade* & $\begin{array}{l}\text { G. A. Damasceno- } \\
\text { Junior } 3580\end{array}$ & $\mathrm{COR}$ & $\begin{array}{l}\text { MR, CE, } \\
\text { FED, FES }\end{array}$ & 1 \\
\hline Sapindaceae & Cardiospermum halicacabum L. & Poca* & V. J. Pott 372 & CPAP & $\begin{array}{l}\text { FES, CI, } \\
\text { RU }\end{array}$ & 1 \\
\hline Sapindaceae & $\begin{array}{l}\text { Allophylus edulis (A. St.-Hil. et } \\
\text { al.) Hieron. ex Niederl. }\end{array}$ & Cuncum & A. Pott 13697 & CGMS & $\mathrm{CE}$ & 6 \\
\hline Sapindaceae & Allophylus pauciflorus Radlk. & Cuncum & A. Pott 9423 & CGMS & FES & 6 \\
\hline Sapindaceae & Dilodendron bipinnatum Radlk. & $\begin{array}{l}\text { Mulher-pobre*, maria- } \\
\text { pobre*, mãe-pobre (1) }\end{array}$ & A. Pott 2953 & CPAP & $\begin{array}{l}\text { MR, CE, } \\
\text { FED, FES }\end{array}$ & 1,2 \\
\hline Sapindaceae & Melicoccus lepidopetalus Radlk. & $\begin{array}{l}\text { Água-pomba*, água- } \\
\text { pomba macho, pitomba } \\
\text { (1), "mapó" (Gt, 24) }\end{array}$ & $\begin{array}{l}\text { I. M. Bortolotto } \\
1053\end{array}$ & CPAP & $\begin{array}{l}\text { MR, CH, } \\
\text { CE, FED, } \\
\text { FES }\end{array}$ & $\begin{array}{c}1,2,3 \\
5,24\end{array}$ \\
\hline Sapindaceae & Paullinia elegans Cambess. & - & A. Pott 4527 & $\mathrm{COR}$ & FES & 1,2 \\
\hline Sapindaceae & Paullinia pinnata $\mathrm{L}$. & $\begin{array}{l}\text { Cipó-cinco-folha, fruta-de- } \\
\text { pomba (1) }\end{array}$ & $\begin{array}{l}\text { G. A. Damasceno- } \\
\text { Junior } 323\end{array}$ & COR & $\begin{array}{l}\text { MR, FES, } \\
\text { CIP }\end{array}$ & 1,2 \\
\hline Sapindaceae & $\begin{array}{c}\text { Talisia esculenta (Cambess) } \\
\text { Radlk. }\end{array}$ & Pitomba* & $\begin{array}{l}\text { I. M. Bortolotto } \\
1227\end{array}$ & $\mathrm{COR}$ & $\begin{array}{l}\text { MR, FED, } \\
\text { FES, RU }\end{array}$ & $\begin{array}{l}3,5,6 \\
7,12\end{array}$ \\
\hline Sapotaceae & $\begin{array}{l}\text { Chrysophyllum gonocarpum } \\
\text { (Mart. \& Eichl. ex Miq.) Engl. }\end{array}$ & - & $\begin{array}{l}\text { G. A. Damasceno- } \\
\text { Junior } 1576\end{array}$ & $\mathrm{COR}$ & MR, FED & 6 \\
\hline Sapotaceae & $\begin{array}{l}\text { Chrysophyllum marginatum } \\
\text { (Hook. \& Arn.) Radlk. }\end{array}$ & $\begin{array}{l}\text { Leiteirinho, uvinha, } \\
\text { pimenteira-de-aranquã (1) }\end{array}$ & $\begin{array}{l}\text { G. A. Damasceno- } \\
\text { Junior } 2492\end{array}$ & $\mathrm{COR}$ & CE, FES, & 1,2 \\
\hline Sapotaceae & $\begin{array}{l}\text { Pouteria gardneri (Mart. \& } \\
\text { Miq.) Baehni }\end{array}$ & Frutinha-de-veado (2) & POTT EL AL. & VER & FES & 2,7 \\
\hline Sapotaceae & $\begin{array}{l}\text { Pouteria glomerata (Mart. \& } \\
\text { Miq.) Baehni. }\end{array}$ & $\begin{array}{l}\text { Laranjinha-de-pacu*, } \\
\text { moranguinha*, parada (1), } \\
\text { laranjinha*, "macondjê" (Gt } \\
\text { 24) }\end{array}$ & $\begin{array}{l}\text { I. M. Bortolotto } \\
911\end{array}$ & $\mathrm{COR}$ & MR & $\begin{array}{c}1,2,5 \\
624\end{array}$ \\
\hline Sapotaceae & $\begin{array}{l}\text { Pouteria ramiflora (Mart.) } \\
\text { Radlk. }\end{array}$ & Fruta-de-veado , fruteira (1) & A. Pott 5453 & CPAP & $\mathrm{CE}$ & $1,2,7$ \\
\hline Sapotaceae & Pouteria torta (Mart.) Radlk. & Laranjinha (1) & $\begin{array}{l}\text { G. A. Damasceno- } \\
\text { Junior } 1912\end{array}$ & COR & $\begin{array}{l}\text { MR, CE, } \\
\text { FES }\end{array}$ & $1,7,24$ \\
\hline Sapotaceae & $\begin{array}{l}\text { Sideroxylon obtusifolium (Roem. } \\
\text { \& Schult.) T.D. Penn. }\end{array}$ & $\begin{array}{l}\text { Guajuviraí (1), laranjinha- } \\
\text { preta }\end{array}$ & A. Pott 4621 & CPAP & $\begin{array}{l}\text { CH, FED, } \\
\text { FES }\end{array}$ & $1,2,7$. \\
\hline Solanaceae & Capsicum baccatum $\mathrm{L}$. & Pimenta* & $\begin{array}{l}\text { G. A. Damasceno- } \\
\text { Junior } 2447\end{array}$ & COR & & 18 \\
\hline Solanaceae & Physalis peruviana $\mathrm{L}$. & - & A. Pott 5490 & CPAP & RU & 1,2 \\
\hline Solanaceae & Solanum lycocarpum A. St.-Hil. & Lobeira & A. Pott 14118 & CGMS & $\mathrm{CE}$ & 6 \\
\hline Solanaceae & Solanum paniculatum $\mathrm{L}$. & Jurubeba* & $\begin{array}{l}\text { G. A. Damasceno- } \\
\text { Junior } 3775\end{array}$ & COR & MR, RU & 2 \\
\hline Talinaceae & $\begin{array}{l}\text { Talinum triangulare (Jacq.) } \\
\text { Willd. }\end{array}$ & Caruru*, alfavaca (1) & A. Pott 2696 & CPAP & FES & 1,2 \\
\hline Typhaceae & Typha domingensis Pers. & Taboa* & A. Pott 4925 & CPAP & CIP & $2,4,19$ \\
\hline Urticaceae & Cecropia pachystachya Trécul & Embaúba*, embauva* & $\begin{array}{l}\text { I. M. Bortolotto } \\
1055\end{array}$ & $\mathrm{COR}$ & $\begin{array}{l}\text { MR, CH, } \\
\text { CE, FED, } \\
\text { FES, CIP, } \\
\text { RU }\end{array}$ & 1,2 \\
\hline Urticaceae & Cecropia saxatilis Snethl. & Embaúba*, Embaúva* & A. Pott 9536 & CGMS & $\mathrm{CE}$ & 6 \\
\hline Urticaceae & Urera aurantiaca Wedd. & $\begin{array}{l}\text { Urtiga-de-pacu, urtiga, } \\
\text { caçanção (1) }\end{array}$ & A. Pott 3563 & CPAP & $\begin{array}{l}\text { MR, FED, } \\
\text { FES, CIP }\end{array}$ & 1,2 \\
\hline Verbenaceae & Lantana trifolia $\mathrm{L}$. & $\begin{array}{l}\text { Cidreira (falsa), uvinha- } \\
\text { do-campo (1) }\end{array}$ & A. Pott 5418 & CPAP & FES & 1 \\
\hline Verbenaceae & $\begin{array}{c}\text { Lippia alba (Mill.) N.E. Br. ex } \\
\text { P. Wilson }\end{array}$ & $\begin{array}{l}\text { Cidreira-do-campo*, chá- } \\
\text { de-zezinho* }\end{array}$ & $\begin{array}{l}\text { I. M. Bortolotto } \\
1491\end{array}$ & $\mathrm{COR}$ & MR & 0 \\
\hline Verbenaceae & Aegiphila verticillata Vell. & Tamanqueria* & A. Pott 13993 & CGMS & $\mathrm{CE}$ & 6 \\
\hline Vitaceae & $\begin{array}{c}\text { Cissus campestris (Baker) } \\
\text { Planch. }\end{array}$ & Cipó-de-arraia (1) & A. Pott 2381 & CPAP & MR & 1,2 \\
\hline
\end{tabular}


Quadro 1. Cont.

\begin{tabular}{|c|c|c|c|c|c|c|}
\hline Família & Nome científico & $\begin{array}{l}\text { Nome popular no MS* } \\
\text { "nome indígena"e (fonte) }\end{array}$ & $\begin{array}{l}\text { Nome e Número } \\
\text { do coletor }\end{array}$ & Herbário & Fisionomia & Fontes \\
\hline Ximeniaceae & Ximenia americana $\mathrm{L}$. & $\begin{array}{l}\text { Limãozinho-do-cerrado, } \\
\text { pessegueira, limão-bravo, } \\
\text { limãozinho (1) }\end{array}$ & A. Pott 1456 & CPAP & CE, FES & 1,2 \\
\hline
\end{tabular}

A família Dioscoreaceae é reconhecidamente importante para fins alimentícios devido à presença de tubérculos ricos em amido, com cerca de 20 espécies citadas como alimentícias no Brasil (Pio Corrêa 1926-1978). Dentre as listadas neste trabalho, destacamos Dioscorea trifida, domesticada pelos índios da América do Sul (Siqueira 2009) e nativa do Brasil (Kirizawa et al. 2015. Essa espécie, juntamente com $D$. dodecaneura e $D$. piperifolia estão entre as mais utilizadas para alimentação humana no Brasil (Pedralli 2002). Siqueira (2009) discute que as pesquisas relacionadas ao melhoramento e à conservação de Dioscoreaceae têm sido negligenciadas. Em Mato Grosso do Sul, estudos voltados ao cultivo de Dioscorea spp. (espécies domesticadas) têm sido desenvolvidos (Heredia Zárate et al. 2000), mas a única referência de uso alimentícios por populações locais foi o de $D$. trifida (Pott e Pott 1994). D. trifida e D. ovata, são citadas pela primeira vez para o Mato Grosso do Sul.

Merecem destaque também duas espécies de jaracatiá: Jacaratia corumbensis e J. spinosa. A primeira tem um sistema subterrâneo com uso conhecido para alimentação, adicionado à rapadura de cana (Saccharum officinarum L.). No estado ela ocorre nas florestas estacionais deciduais do Pantanal e é pouco aproveitada, mas como tem potencial alimentício, o estímulo ao uso deve ser acompanhado de uma política de manejo conservacionista. O mesmo ocorre para J. spinosa cuja medula do caule aéreo é aproveitada para produção de doces com fins comerciais (comercializada no município de Bonito). Damasceno-Junior et al. (2010) fazem a mesma recomendação para Victoria amazonica, cujas sementes podem ser aproveitadas na dieta, mas tem ocorrência restrita e em pequenas populações.

Cynophalla retusa ocorre no Chaco brasileiro (município de Porto Murtinho) e ainda não tem registro sobre sua ocorrência em outras fitofisionomias do Brasil. Seu uso alimentício foi mencionado apenas em estudos etnobotânicos desenvolvidos com indígenas nos países vizinhos, como Arenas \& Scarpa (2007) na Argentina com os Charote e por Susnik (1982) no Paraguai com os Chamacoco. Da mesma forma, Capparicordis tweediana não está na Lista de Espécies da Flora do Brasil (2012) para o Brasil ou MS e tem registro de uso alimentício citado apenas para Argentina (Arenas \& Scarpa 2007) e Bolívia (UMSA et al. 2002). Capparidastrum coimbranum, que foi descrita em 2005 (Cornejo \& Iltis 2005) para a Bolívia, é citada pela primeira vez como comestível para a literatura científica. Possui frutos com polpa saborosa e suavemente adocicada.

Dioscorea aesculifolia foi citada para o Mato Grosso do Sul na Lista de Espécies da Flora do Brasil (Kirizawa et al. 2015), e consta como vulnerável na lista oficial de espécies de plantas ameaçadas de extinção no estado de São Paulo (Resolução SMA 48 2004). Mauritia flexuosa, amplamente aproveitada para diversos fins, inclusive o alimentício (Santos \& Coelho-Ferreira 2012), ocorre em áreas de Veredas, consideradas protegidas pelo código florestal, mas ainda é grande a descaracterização dos habitats dessa espécie no estado.

As fisionomias com maior número de espécies foram o Cerrado (137), a Mata Ribeirinha (103), a Floresta Estacional Semidecidual (80), a Floresta Estacional Decidual (45) e os Campos Inundáveis no Pantanal (42), seguidas pelo Chaco (19) e pelos Campos inundáveis fora do Pantanal, que inclui as Veredas (12). Muitas dessas fisionomias no MS estão sob forte pressão antrópica devido à expansão das fronteiras agrícolas, sobretudo as áreas de Cerrado e Florestas Estacionais (Silva et al .2011). Apenas quatro espécies foram coletadas de áreas de cultivo e 12 são ruderais.

As espécies que ocorrem como formações monodominantes têm denominação específica, sobretudo no Pantanal, como o "arrozal" formada por Oryza spp., o carandazal (Copernicia alba), o bocaiuval (Acrocomia aculeata), o canjiqueiral (Byrsonima cydoniifolia) e o babaçual (Attalea speciosa). Essas formações em geral apresentam mais de $50 \%$ dos indivíduos ou da cobertura formada por uma única espécie (Connell \& Lowman 1989) e são, em muitos casos, consideradas "invasoras" (Pott \& Pott 2000a). Attalea phalerata, por exemplo, cujos frutos têm potencial alimentício (Pio Corrêa 1926-1978) é considerada invasora de pastagens no Cerrado (Pott \& Pott 2000 a) e em muitas propriedades rurais que se dedicam à criação de gado bovino os indivíduos são eliminados. Pelo fato de ocorrerem como formações relativamente homogêneas e ainda com grande produção de frutos, se constituem em uma excelente oportunidade para propostas de aproveitamento para fins alimentícios com manejo sustentável, sem necessidade de modificação dos ambientes envolvidos e com possibilidade de produção em larga escala.

O Mato Grosso do Sul detém populações nativas de espécies cultivadas ou de parentes próximas de espécies cultivadas com interesse econômico, que fazem parte da dieta humana no mundo todo, como comentamos acima a respeito de Arachis. O Brasil é uma grande fonte de diversidade genética de espécies de Arachis (Valls 2005) e o Mato Grosso do Sul tem responsabilidade na conservação in situ e na investigação científica sobre esse importante recurso. Destacamos aqui Stevia rebaudiana usada há séculos pelos índios Guarani (Lewis 1992) como adoçante para chá 
mate (preparado com folhas de Ilex paraguariensis) e pela indústria de alimentos como um substituto não calórico para o açúcar. Oryza glumaepatula e O. latifolia associados à cultura Guató (Oliveira 1996) têm populações protegidas no Parque Nacional do Pantanal Mato-Grossense (MT), mas não há registro de áreas protegidas ou políticas públicas voltadas à conservação in situ dos recursos genéticos no Mato Grosso do Sul. Em função da grande diversidade de recursos genéticos estratégicos para o futuro, o Mato Grosso do Sul precisa adotar, dentre os critérios para criação e manutenção de unidades de conservação, a presença de espécies alimentícias ou com potencial alimentício para a dieta humana. Como esses recursos são estratégicos e envolvem a segurança alimentar, urge a criação de novas unidades de conservação de uso sustentável e ainda a elaboração de políticas que visem a conservação desses recursos in situ de forma a manter o seu potencial e ainda a sua variabilidade genética.

\section{AGRADECIMENTOS}

Aos Herbários COR, CPAP e CGMS e ao INCT. Aos especialistas que colaboraram com os autores e identificaram ou confirmaram a identificação das espécies: Armando Cervi (Passiflora) in memoriam, Ângela Lúcia Bagnatori Sartori (Fabaceae), Jimi Nakajima (Asteraceae), Mizue Kirizawa (Dioscorea) e Mônica Morales (Arecaceae).

\section{REFERÊNCIAS}

Amaral, C. N. do \& Guarim Neto, G. 2008. Os quintais como espaços de conservação e cultivo de alimentos: um estudo na cidade de Rosário Oeste (Mato Grosso, Brasil) Boletim do Museu Paraense Emílio Goeldi. Ciências Humanas 3 (3): 329-341.

Arenas, P. \& Scarpa, G. F. 2007. Edible wild plants of the Chorote Indians, Gran Chaco, Argentina. Botanical Journal of the Linnean Society $153: 73-85$.

Berg, M. E. Van Den. 1986. Formas atuais e potenciais de aproveitamento das espécies nativas e exóticas do Pantanal Mato-grossense. In Anais do I Simpósio Sobre Recursos Naturais e Sócio-Econômicos do Pantanal, 1984. Empresa Brasileira de Pesquisa Agropecuária, Brasília, p. 131-136.

Bezerra, J. E. F., Lederman, I. E. Silva Junior, J. F. da \& Proença, C. E. B. 2006. Araçá. In Frutas nativas da região Centro-Oeste do Brasil (R. F. Vieira ed.). Empresa Brasileira de Pesquisa Agropecuária. Recursos Genéticos e Biotecnologia, Brasília, p.153-161.

Bortolotto, I. M. \& Amorozo, M. C. M. 2012. Aspectos históricos e estratégias de subsistência nas comunidades localizadas ao longo do rio Paraguai em Corumbá - MS. In Pantanal: territorialidades, culturas e diversidade (E. C. Moretti. E. C. \& A. Banducci Junior eds.). Editora Universidade Federal de Mato Grosso do Sul, Campo Grande, p. $57-88$.

Bortolotto, I. M., Amorozo, M. C. M., Guarim Neto G., Oldeland J. \& Damasceno-Junior, G. A. 2015. Use of wild edible plants in rural communities along Paraguay River, Pantanal, Brazil. Journal of Ethnobiology and Ethnomedicine 11(46): $1-14$

Cabeza de Vaca, Á. N.1987. Naufrágios e comentários. Trad. Jurandir Soares dos Santos. L \& PM, Porto Alegre. 240 p.

Conceição, C. de A. \& Paula, J. E. de 1986. Contribuição para o conhecimento da flora do Pantanal mato-grossense e sua relação com a fauna e o homem. In Anais do I Simpósio Sobre Recursos Naturais e Sócio-Econômicos do Pantanal, 1984. Empresa Brasileira de Pesquisa Agropecuária, Brasília, p. 107-136.

1990. Contribuição para o conhecimento da flora do Pantanal mato-grossense. Revista Científica e Cultural 5(1):13 - 22.
Connell, J.H. \& Lowman, M.D. 1989. Low-diversity tropical rain forests: some possible mechanisms for their existence. American Naturalist 134(1):88-119.

Cornejo, S.X. \& Iltis, H. H. 2005. Studies in the Capparaceae XXIII: Capparis coimbrana, a new species from Bolivia. Brittonia 57(2):155-161.

Costa. M. de F. 1999. História de um país inexistente. O Pantanal entre os séculos XVI e XVIII. Kosmos, São Paulo. 277 p.

Damasceno-Junior, G. A., Souza, P. R. Bortolotto, I. M., Ramos, M. I. L., Hiane, P. A., Braga Neto, J. A., Ishii, I. H., Costa, D.C., Ramos Filho, M.M., Gomes, R. J. B., Barbosa \& M. M. Rodrigues, R. B. 2010. Sabores do Cerrado e Pantanal: conhecer para valorizar os frutos nativos; receitas e boas práticas de aproveitamento. Editora da Universidade Federal de Mato Grosso do Sul, Campo Grande. 142p.

Franco, J.L.A. \& Drummond, J.A. 2005. Frederico Carlos Hoehne: a atualidade de um pioneiro no Campo da proteção à natureza do Brasil, Ambiente e Sociedade. Disponível em: http://www.scielo. $\mathrm{br} / \mathrm{pdf} / \mathrm{asoc} / \mathrm{v} 8 \mathrm{n} 1 / \mathrm{a} 09 \mathrm{v} 08 \mathrm{n} 1$. Acessado em 25.3.2015.

Haynes, J. \& McLaughlin, J.2000. Edible Palms and Their Uses. Institute of Food and Agricultural Science.University of Florida Cooperative Extension Service.13 p. Disponível em: http://www.plantapalm. com/vpe/ethnobotany/EdiblePalms.PDF. Acessado em 15.11.2012.

Herberts, A. L. 1998. Os Mbayá-Guaicurú: área, assentamento, subsistência e cultura material. 1998. Dissertação, Instituto Anchietano de Pesquisas, Universidade do Vale do Rio dos Sinos, São Leopoldo.

Heredia Zárate, N. A., Vieira, M. C. \& Minuzzi, A. 2000. Produção de cará (Dioscorea sp.) em diferentes densidades de plantio. Ciência e Agrotecnologia 24(2):387-391.

Hoehne, F. C. 1946. Frutas indígenas. São Paulo. Instituto de Botânica (Publicação da série D). Secretaria da Agricultura, Indústria e Comércio, São Paulo. 88 p.

Instituto Nacional de Ciência e Tecnologia-INCT. Herbário Virtual da Flora e dos Fungos. Disponível em: http://lacunas.inct.florabrasil. net/index. Acessado em 15.11.2012.

Judd, W.S., Campbell, C.S., Kellogg, E.A. \& Stevens, P.F. 1999. Plant systematics: a phylogenetic approach. Sunderland. Sinauer Associates. 462p.

Kinupp, V.F. 2007. Plantas alimentícias não-convencionais da Região Metropolitana de Porto Alegre, RS. Tese 562 f., Faculdade de Agronomia. Universidade do Rio Grande do Sul, Porto Alegre, RS.

Kinupp,V.F. , Barros, I.B.I. 2004. Levantamento de dados e potencial de plantas alimentícias alternativas no Brasil. Horticultura Brasileira v. 22., n 2. Disponível em: http://www.esalq.usp.br/siesalq/pm/ plantas alimenticias.pdf. Acessado em 02.11.2013.

Kirizawa, M, Xifreda, C.C., Couto, R., Araújo, D. 2015. Dioscoreaceae In Lista de Espécies da Flora do Brasil. Jardim Botânico do Rio de Janeiro. Disponível em: <http://floradobrasil. jbrj.gov.br/jabot/floradobrasil/FB17306>. Acessado em 25.3. 2015.

Kunkel, G. 1984. Plants for Human consumption. Koenigstten. Koeltz Scientific Books. 393p.

Leite, M. L. M. Naturalistas viajantes. 1995. História, Ciências, Saúde. Manguinhos, vol.1, n.2, pp. 7-19. Disponível em: http://www.scielo. $\mathrm{br} / \mathrm{pdf} / \mathrm{hcsm} / \mathrm{v} 1 \mathrm{n} 2 / \mathrm{a} 02 \mathrm{v} 1 \mathrm{n} 2$.pdf. Acessado em 15.11.2012.

Lewis, W. H. 1992. Early uses of Stevia rebaudiana (Asteraceae) leaves as a sweetener in Paraguay. Economic Botany 46: 336-337. Disponível em: http://dx.doi.org/10.1007/BF02866633. Acessado em 15.11.2012.

Lista de Espécies da Flora do Brasil 2013. Jardim Botânico do Rio de Janeiro. Disponível em: http://floradobrasil.jbrj.gov.br/. Acessado em 15.11.2012

Lorenzi, H., Bacher, L., Lacerda, M.\& Sartori, S. 2006. Frutas brasileiras e exóticas cultivadas: de consumo in natura. Instituto Plantarum de Estudos da Flora, São Paulo. 640 p.

Lorenzi, H., Noblick, L., Kahn, F. \& Ferreira, E. 2010. Flora Brasileira Lorenzi Arecaceae (palmeiras). Instituto Plantarum. Nova Odessa. $368 \mathrm{p}$.

Martins, M. R., Oliveira, J. C. de, Di Mauro, A. O. \& Silva, P. C. da 2003. Avaliação de populações de maracujazeiro-doce (Passiflora alata Curtis) obtidas de polinização aberta. Rev. Bras. Frutic. [online] 25 (1): 111-114. Disponível em: http://dx.doi.org/10.1590/S010029452003000100032. Acessado em 15.11.2012. 
Ministério da Saúde. 2002. Alimentos regionais brasileiros. Disponível em: http://189.28.128.100/nutricao/docs/geral/alimentos_regionais_ brasileiros.pdf. Acessado em 15.11.2012.

Mostacedo, B. C. \& Uslar, Y. J. 1999. Plantas silvestres con frutos y semillas comestibles del departamento de Santa Cruz, Bolivia: un inventario preliminar. Revista de la Sociedad Boliviana de Botánica 2(2): 203-226

Oliveira, J. E. de 1996. Guató: argonautas do pantanal. Editora Universitária da Pontifícia Universidade Católica do Rio Grande do Sul. Porto Alegre. 179 p.

Pedralli, G. 2002. Dioscoreaceae e Araceae: aspectos taxonômicos, etnobotânicos e espécies nativas com potencial para melhoramento genético. In Santos, E. S. (Ed). Simpósio Nacional sobre as Culturas do Inhame e do Taro, João Pessoa. p. 37-53.

Pennington, T.D. 1997. The Genus Inga. Botany. Royal Botanical Garden, Kew, 844 p.

Pio Corrêa, M. 1926-1978. Dicionário das plantas úteis do Brasil e das exóticas cultivadas. Imprensa Nacional, Rio de Janeiro. 540p.

Pott, A., Oliveira, A.K.M., Damasceno-Junior, G.A., Silva. J.S.V. 2011. Plant diversity of the Brazilian Pantanal wetland. Brazilian Journal of Biology 71(1): 265-273.

Pott, A \& Pott, V.J. 1994. Plantas do Pantanal. Empresa Brasileira de Pesquisa Agropecuária, Brasília. 320 p.

.V. J. 2000 a. Lista preliminar de plantas invasoras atuais e potenciais de pastagens do Centro Oeste. [S. 1.: s. n.]. 16 p. .2000 b. Plantas Aquáticas do Pantanal. Empresa Brasileira de Pesquisa Agropecuária, Brasília. 404p

Pott, A., Pott, V. J. \& Sobrinho, A. A. B. 2004. Plantas úteis à sobrevivência no Pantanal. In Anais do IV Simpósio sobre recursos Naturais e Sócio econômicos do Pantanal. Empresa Brasileira de Pesquisa Agropecuária, Corumbá. p.81-92.

Ramos, M. I. Lima, Ramos Filho, M. M., Hiane, P. A., Braga Neto, J. A., \& Siqueira, E. M. de A. 2008. Qualidade nutricional da polpa de bocaiúva Acrocomia aculeata (Jacq.) Lodd. Ciência e Tecnologia de Alimentos, 28 (Suppl. ), 90-94. Disponível em: http://dx.doi. org/10.1590/S0101-20612008000500015. Acessado em 15.11.2012.
Resolução Secretaria de Meio Ambiente 48. 2004 Lista oficial das espécies da flora do Estado de São Paulo ameaçadas de extinção. Disponível em: http://botanica.sp.gov.br/files/2014/02/resolu\%C3\%A7\%C3\%A3o_sma48.pdf. Acessado em 29.04.2015.

Santos, R. da S. \& Coelho-Ferreira, M. 2012. Estudo etnobotânico de Mauritia flexuosa L. f. (Arecaceae) em comunidades ribeirinhas do Município de Abaetetuba, Pará, Brasil. Acta Amazonica, Manaus, v. 42, n. 1, Mar. 2012 . Disponível em: http://dx.doi.org/10.1590/ S0044-59672012000100001. Acessado em 15.11.2012.

Silva, J.S.V., Pott, A., Abdon, M.M., Pott, V.J. \& Santos, K.R. 2011. Projeto GeoMS: cobertura vegetal e uso da terra do Estado de Mato Grosso do Sul. Empresa Brasileira de Pesquisa Agropecuária. Informática Agropecuária, Campinas. 64 p.

Siqueira, M.V.B. M. 2009. Inhame (Dioscorea spp.): uma cultura ainda negligenciada. Horticultura Brasileira 27: S4075-S4090. Disponível em: http:/www.abhorticultura.com.br/eventosx/trabalhos/ev_3/P_20_ Artigo Palestra Marcos Vinicius.pdf. Acessado em 15.11.2012.

Soares, J. de M. 2009. Etnobotânica de Syagrus oleracea (Mart.) Becc. (Arecaceae) em Campo Grande, Mato Grosso do Sul, Brasil. Monografia 16 f. Universidade Federal de Mato Grosso do Sul, Campo Grande.

Susnik, B. 1982. Los Aborigenes del Paraguay. IV Cultura Material. Museo Etnográfico Andres Barbero, Asunción. 237 p.

Universidad Mayor de San Andrés - UMSA, Fundación Kaa-Iya, Instituto de Investigaciones para el Desarrollo, Capitania Del Alto Y Bajo Izozog, Wildlife Conservation Society (Bolivia), Herbario Nacional de Bolivia, Programa de Ciencia y Tecnología para el Desarrollo \& Organización de Estados Americanos (Eds.). 2002. Plantas del Chaco II: usos tradicionales Izoceño-Guaraní. Santa Cruz, 441 p. Disponível em: <http://horizon.documentation.ird.fr/exl-doc/pleins_textes/ divers10-04/010029501.pdf.>. Acesso em 15.11.2012.

Valls, J.F.M. 2005. Recursos genéticos de Arachis: Avanços no conhecimento botânico e a situação atual de conservação e uso. Agrociencia 9(1-2):123-132.

. 2012. Arachis In Lista de Espécies da Flora do Brasil. Jardim Botânico do Rio de Janeiro. Disponível em http://floradobrasil.jbrj. gov.br/2012/FB022798. Acessado em 15/11/2012. 